\title{
An a posteriori-implicit turbulent model with automatic dissipation adjustment for Large Eddy Simulation of compressible flows
}

\author{
Xesús Nogueira ${ }^{\mathrm{a}, *}$, Luis Ramírez ${ }^{\mathrm{a}}$, Javier Fernández-Fidalgo ${ }^{\mathrm{a}}$, Michael \\ Deligant $^{\mathrm{b}}$, Sofiane Khelladi ${ }^{\mathrm{b}}$, Jean-Camille Chassaing ${ }^{\mathrm{c}}$, Fermín Navarrina ${ }^{\mathrm{a}}$ \\ ${ }^{a}$ Universidade da Coruña,Group of Numerical Methods in Engineering, Campus de \\ Elviña, 15071, A Coruña, Spain \\ ${ }^{b}$ Laboratoire de Dynamique des Fluides, Arts et Métiers ParisTech, 151 Boulevard de \\ l'Hôpital. 75013 Paris, France. \\ ${ }^{c}$ Sorbonne Université, UPMC Univ Paris 06, CNRS, UMR7190, D'Alembert Institute, \\ F-75005 Paris, France
}

\begin{abstract}
In this work we present an a posteriori high-order finite volume scheme for the computation of compressible turbulent flows. An automatic dissipation adjustment (ADA) method is combined with the a posteriori paradigm, in order to obtain an implicit subgrid scale model and preserve the stability of the numerical method. Thus, the numerical scheme is designed to increase the dissipation in the control volumes where the flow is under-resolved, and to decrease the dissipation in those cells where there is excessive dissipation. This is achieved by adding a multiplicative factor to the dissipative part of the numerical flux. In order to keep the stability of the numerical scheme, the a posteriori approach is used. It allows to increase the dissipation quickly in cells near shocks if required, ensuring the stability of the scheme. Some numerical tests are performed to highlight the accuracy and robustness of
\end{abstract}

\footnotetext{
*Corresponding author, e-mail: xesus.nogueira@udc.es
} 
the proposed numerical scheme.

Keywords: High-order methods, Compressible flows, Computational Fluid

Dynamics, Implicit Large Eddy Simulation, A posteriori shock capturing

\section{Introduction}

Compressible turbulent flows are important in many scientific and engineering applications, such as scramjet propulsion, supersonic aircraft design, high-temperature reactive flows, inertial confinement fusion, and starforming clouds in galaxies. In turbulent flows of engineering interest, it is not suitable to simulate the complete range of scales that are present in the flow, since the number of degrees of freedom of turbulence grows with the Reynolds number faster than $\mathcal{O}\left(R e^{11 / 4}\right)[1]$. This huge number of degrees of freedom, is far out of the possibilities of computation using current computers and this situation is expected to continue, at least, for the next decades. In this context, Large Eddy Simulation (LES) is the most suitable approach for the computation of these flows. However, the numerical simulation of compressible turbulent flows is a very challenging task, due to the complexity of the involved physical processes. Since LES simulations do not solve the complete range of scales of the flow, the effect of the unresolved scales should be adequately modeled in order to get the most accurate solution on a given grid. This is the reason for the existence of the so-called subgrid scale (SGS) models. The development of SGS models is a very active area of research. However, most of the subgrid models existing in the literature have been developed for incompressible flows, and they do not account for the intermodal energy transfer which takes place in compressible turbulence 
[1]. The physical mechanism driving this transfer is completely different to the corresponding one to the interscale energy transfer, which is the basis of SGS models for incompressible flows. Yet another problem of the simulation of turbulent compressible flows is the possible presence of shocks, that makes mandatory the use of stabilization methods, which may introduce additional dissipation $[2,3,4]$. Thus, the main issue is how to introduce the right amount of numerical dissipation for stabilization with minimal interference in vortical dynamics. Moreover, the accuracy of a LES computation does not depend on only one aspect, but it is also dependent on the combination and coupling of several factors, as the discretization scheme (time and spatial), the resolution and quality of the grid and the SGS model (if used). In particular, the dissipation of the numerical scheme is a key feature that determines if a given numerical method is suitable for LES computations. In this context, Implicit Large Eddy Simulation (ILES) proposes to use the truncation error of discretization schemes for modeling the effect of subgrid scales on resolved scales $[5,6,7,8,9]$. Thus, in ILES computations, the numerical scheme plays implicitly the role of the SGS model. However, not all the schemes are suitable for ILES computations [10]. Standard second-order schemes are over-dissipative, and thus, they are not well suited for LES (nor ILES) computations. Higher-order approximations are an alternative [11, 12, 13, 14], but a number of authors have noted that the discretization scheme needs to be designed specifically for ILES in order to obtain better results than explicit SGS models [15]. In [16, 17], in the framework of the Adaptive Local Deconvolution Method (ALDM), the numerical viscosity of the method is optimized in order to minimize the difference with the spectral eddy vis- 
cosity of Eddy Damped Quasi-Normal Markovian (EDQNM) theory. Other approaches have also been presented in the literature for DNS and LES computations. Hybrid schemes that blend a high order non-dissipative scheme and high order shock-capturing have been developed based on the different nature of turbulence and shock waves [18]. A shock sensor based on vorticity and dilatation is used for switching to one of the different schemes. Yet a different approach is to use a non-dissipative scheme as a base scheme and then a post-processing step to nonlinearly filtering the solution by a dissipative portion of a high-order shock-capturing scheme with a local flow sensor $[19,20,21]$.

Finite volumes (FV) are the most commonly used methods for the simulation of compressible flows. These methods are based on the use of Riemann solvers [22]. In this context, the Roe scheme[23] is one of the numerical fluxes which is most widely used for compressible flow computations. If some corrections are included in the original formulation [4, 24, 25], it is also suitable for low Mach computations.

In a number of contributions [26, 27], self-adaptive upwind methods are proposed to reduce the dissipation introduced by the Roe scheme as much as possible. In [28] an Automatic Dissipation Adjustment method (ADA) for low Mach computations using the LMRoe scheme was presented. In this method, the energy ratio (ER) criteria [29] is used to automatically adjust the amount of viscosity introduced by the numerical flux. In this work, we aim to extend the ADA method for the computation of compressible flows with shock waves. In order to address the problem of stabilization due to shocks we propose to use the a posteriori paradigm [30, 31]. The ER 
criteria adjusts the dissipation in the smooth zones of the flow, whereas the a posteriori paradigm preserves the stability by increasing the dissipation if the computations fail or spurious oscillations appear. Thus, in this work, we determine numerically if the scheme should introduce more dissipation or not, avoiding the use of specific sensors to discern between turbulent fluctuations or shocks. The results obtained show a great accuracy improvement in terms of the distribution of energy in the wavenumber spectrum as well as in the decay of kinetic energy, and thermodynamic variables. The only constraint of the numerical flux in order to apply the proposed methodology is that it must be possible to write the flux as a sum of a central part and a dissipation part. In this work we show the applicability of the proposed methodology to the numerical fluxes of Roe and Rusanov.

Note that in this work we have used a numerical scheme which is thirdorder of accuracy in space and in time. Most of the state-of-the-art methods for DNS and LES present a higher order of accuracy [18, 21, 32, 33]. Moreover, most of these methods are developed for structured grids. However, in industrial applications of compressible CFD, second or third order finite volume methods are the standard. One of the objectives of this work is to show that using the proposed methodology with a third-order scheme it is possible to obtain results comparable to those obtained using very highorder schemes. In particular, we will show that using the proposed scheme the physics of the turbulent decay phenomena is reproduced.

The paper is structured as follows. In section 2 the governing equations are presented. In section 3 the formulation of the proposed methodology is exposed, and in section 4 some numerical tests are presented to show the ac- 
curacy and robustness of the proposed methodology. Finally, the conclusions are drawn.

\section{Governing equations}

The 3D Navier-Stokes equations written in non-dimensional variables and expressed in conservative form read as

$$
\frac{\partial \boldsymbol{U}}{\partial \tau}+\frac{\partial \boldsymbol{F}_{x}}{\partial x}+\frac{\partial \boldsymbol{F}_{y}}{\partial y}+\frac{\partial \boldsymbol{F}_{z}}{\partial z}=\frac{1}{R e}\left[\frac{\partial \boldsymbol{F}_{x}^{V}}{\partial x}+\frac{\partial \boldsymbol{F}_{y}^{V}}{\partial y}+\frac{\partial \boldsymbol{F}_{z}^{V}}{\partial z}\right]
$$

with

$\boldsymbol{U}=\left(\begin{array}{c}\rho \\ \rho u \\ \rho v \\ \rho w \\ \rho E\end{array}\right) \quad \boldsymbol{F}_{x}=\left(\begin{array}{c}\rho u \\ \rho u^{2}+p \\ \rho u v \\ \rho u w \\ \rho u H\end{array}\right) \quad \boldsymbol{F}_{y}=\left(\begin{array}{c}\rho v \\ \rho u v \\ \rho v^{2}+p \\ \rho v w \\ \rho v H\end{array}\right) \quad \boldsymbol{F}_{z}=\left(\begin{array}{c}\rho w \\ \rho u w \\ \rho v w \\ \rho w^{2}+p \\ \rho w H\end{array}\right)$

$$
\boldsymbol{F}_{x}^{V}=\left(\begin{array}{c}
0 \\
\tau_{x x} \\
\tau_{x y} \\
\tau_{x z} \\
u \tau_{x x}+v \tau_{x y}+w \tau_{x z}-q_{x}
\end{array}\right)\left(\begin{array}{c}
0 \\
\tau_{x y} \\
\tau_{y y} \\
\tau_{y z} \\
u \tau_{x y}+v \tau_{y y}+w \tau_{y z}-q_{y}
\end{array}\right)
$$




$$
\begin{array}{r}
\rho E=\rho e+\frac{1}{2} \rho(\boldsymbol{v} \cdot \boldsymbol{v}) \\
H=E+\frac{p}{\rho}
\end{array}
$$

where $\rho$ is the density, $\boldsymbol{v}=(u, v, w)$ is the velocity, $\mu$ is the effective viscosity of the fluid, $H$ is the enthalpy, $E$ is the total energy, $e$ is the internal energy and $\rho$ is the density. The viscous stress tensor is defined as

$$
\begin{aligned}
\tau_{x x} & =2 \mu \frac{\partial u}{\partial x}-\frac{2}{3} \mu\left(\frac{\partial u}{\partial x}+\frac{\partial v}{\partial y}+\frac{\partial w}{\partial z}\right) \\
\tau_{y y} & =2 \mu \frac{\partial v}{\partial y}-\frac{2}{3} \mu\left(\frac{\partial u}{\partial x}+\frac{\partial v}{\partial y}+\frac{\partial w}{\partial z}\right) \\
\tau_{z z} & =2 \mu \frac{\partial w}{\partial z}-\frac{2}{3} \mu\left(\frac{\partial u}{\partial x}+\frac{\partial v}{\partial y}+\frac{\partial w}{\partial z}\right) \\
\tau_{x y} & =\mu\left(\frac{\partial u}{\partial y}+\frac{\partial v}{\partial x}\right) \\
\tau_{x z} & =\mu\left(\frac{\partial u}{\partial z}+\frac{\partial w}{\partial x}\right) \\
\tau_{y z} & =\mu\left(\frac{\partial v}{\partial z}+\frac{\partial w}{\partial y}\right)
\end{aligned}
$$

Using this form of the equations, the important flow parameters are the Reynolds number $(R e)$ and the Mach number $\left(M_{a}\right)$. In order to determine the pressure and temperature we use the following non-dimensional ideal-gas EOS

$$
p=(\gamma-1) \rho e=R \rho T
$$

where $R=1 /\left(\gamma M_{a}^{2}\right)$ is the non-dimensional gas constant, with $\gamma=c_{p} / c_{v}$. The speed of sound is computed as $c=\sqrt{\gamma p / \rho}$. We assume that the viscosity depends on the temperature following a power law

$$
\mu(T)=T^{0.75}
$$


Thermal flux $\boldsymbol{q}=\left(q_{x}, q_{y}, q_{z}\right)$, is computed using Fourier's law

$$
q_{x}=-\lambda \frac{\partial T}{\partial x} \quad q_{y}=-\lambda \frac{\partial T}{\partial y} \quad q_{z}=-\lambda \frac{\partial T}{\partial z}
$$

where $\lambda$ is the non-dimensional thermal conductivity defined as

$$
\lambda=\frac{\mu(T)}{(\gamma-1) M_{a}^{2} P r}
$$

We have used a value of the Prandtl number $\operatorname{Pr}=0.72$. Note that no explicit SGS model is used throughout this work.

\section{Numerical method}

In this work we propose a new methodology for the computation of compressible turbulent flows. The new methodology is based on two key aspects. On one hand, the Automatic Dissipation Adjustment (ADA) model [28] that automatically adjust the amount of numerical dissipation following a criterium based on the Energy Ratio [29]. On the other hand, the a posteriori paradigm [30, 31] which allow us to identify the problematic points where the numerical viscosity is not large enough to avoid oscillations. It is important to remark that the ADA method adjusts the numerical dissipation as an

implicit SGS model [28], whereas the a posteriori approach ensures the stability of the numerical method. In all the computations of the present work, the third-order FV-MLS method [34, 35, 36, 37], and we have applied the proposed methodology to both Roe [23] and Rusanov [38] numerical fluxes. However, the proposed methodology is applicable to any other numerical method based on Riemann solvers provided that the numerical flux could be written combining central differencing of the non-linear inviscid fluxes with a smoothing term. 
The integral form of the Navier-Stokes system (1) for each control volume $I$ is

$$
\int_{\Omega_{I}} \frac{\partial \boldsymbol{U}}{\partial t} d \Omega+\int_{\Gamma_{I}}\left(\mathcal{F}^{H}+\mathcal{F}^{V}\right) \cdot \boldsymbol{n} d \Gamma=\int_{\Omega_{I}} \boldsymbol{S} d \Omega
$$

where $\Omega_{I}$ is the volume of the control volume, $\Gamma_{I}$ is the area of the control volume cells and $\boldsymbol{n}=\left(n_{x}, n_{y}, n_{z}\right)^{T}$ is the unitary exterior normal of the contour. $\boldsymbol{U}$ is the vector of variables, $\mathcal{F}^{H}=\left(F_{x}, F_{y}, F_{z}\right)$ and $\mathcal{F}^{V}=\left(F_{x}^{V}, F_{y}^{V}, F_{z}^{V}\right)$ is the viscous flux vector.

Equation (11) can be written in semi-discrete form as

$$
\int_{\Omega_{I}} \frac{\partial \boldsymbol{U}^{h}}{\partial t} d \Omega+\int_{\Gamma_{I}} \boldsymbol{\Theta}\left(\boldsymbol{u}^{h b+}, \boldsymbol{u}^{h b-}\right) d \Gamma+\int_{\Gamma_{I}} \mathcal{F}^{h V} \cdot \boldsymbol{n} d \Gamma=\int_{\Omega_{I}} \boldsymbol{S}\left(\boldsymbol{u}^{h}\right) d \Omega
$$

where $\boldsymbol{\Theta}\left(\boldsymbol{u}^{h b+}, \boldsymbol{u}^{h b-}\right)$ is a suitable numerical flux, and + and - refers to the left and right states of the cell $I$.

In this work we use an explicit Runge-Kutta (RK) schemes for time integration, as indicated in section 4.

\subsection{Automatic Dissipation Adjustment method}

The ADA method was recently developed as an implicit SGS model, and it was applied for the computation of low mach flows [28]. It is based on the local Energy Ratio (ER) introduced by Tantikul and Domaradzki [29] in the context of the Truncated Navier-Stokes (TNS) procedure [39]. In this work, we aim to extend its range of application to all range of Mach number flows.

The ADA method uses a multiplicative coefficient to the dissipation part of the numerical flux of the Riemann solver. For example, in the case of the 
numerical flux of Roe, it can be written as a central flux plus a dissipation part as

$$
\boldsymbol{\Theta}_{i j}=\frac{1}{2}\left(\mathcal{F}^{h H+}+\mathcal{F}^{h H-}\right) \cdot \boldsymbol{n}-\frac{1}{2} \sum_{k=1}^{4} \tilde{\alpha}_{k}\left|\tilde{\lambda}_{k}\right| \tilde{\boldsymbol{r}}_{k}
$$

In equation (13) $\Theta_{i j}$ is the numerical flux at the interface between cells $i$ and $j, \tilde{\lambda}_{k}$ and $\tilde{\boldsymbol{r}}_{k}$ are the eigenvalues and eigenvectors of the approximated Jacobian [23], and $\alpha_{k}$ are the wave strengths. For the sake of brevity, we refer the interested reader to [23, 40] for finding the analytic expressions of these quantities.

Thus, we introduce a coefficient $\epsilon$ to adjust the dissipation added by the numerical flux

$$
\boldsymbol{\Theta}_{i j}=\frac{1}{2}\left(\mathcal{F}^{h H+}+\mathcal{F}^{h H-}\right) \cdot \boldsymbol{n}-\frac{1}{2} \epsilon_{i j} \sum_{k=1}^{4} \tilde{\alpha}_{k}\left|\tilde{\lambda}_{k}\right| \tilde{\boldsymbol{r}}_{k}
$$

This technique can be applied to any other Riemann solver if it can be expressed as a sum of a central flux plus a dissipation part. In this work, we also apply the proposed algorithm to the numerical flux of Rusanov [38]. The expression used for this numerical flux is

$$
\boldsymbol{\Theta}_{i j}=\frac{1}{2}\left(\mathcal{F}^{h H+}+\mathcal{F}^{h H-}\right) \cdot \boldsymbol{n}-\epsilon_{i j} \frac{1}{2} S^{+} \Delta(\boldsymbol{U})
$$

with

$$
S^{+}=\max \left(\left|\boldsymbol{v}^{+}\right|+c^{+},\left|\boldsymbol{v}^{-}\right|+c^{-}\right)
$$

In equation (16) $c$ is the sound velocity and $|\boldsymbol{v}|$ is the modulus of the velocity vector at integration point and $\Delta(\boldsymbol{U})=\left(\boldsymbol{U}^{+}-\boldsymbol{U}^{-}\right)$.

Such approach to reduce the dissipation of the Riemann solver has been proposed by several authors [26, 27]. The key idea of the ADA method is to link the reduction of the dissipation part with the Energy Ratio (ER) [29] 


$$
E R=\frac{\sum_{i=1}^{3}\left(u_{i}-\widetilde{u}_{i}\right)^{2}}{\sum_{i=1}^{3}\left(u_{i}-\widehat{u}_{i}\right)^{2}}
$$

In equation (17), $u_{i}$ is the velocity field obtained as a result of the computations to solve the Navier-Stokes equation on a given grid using the Riemann solver. Moreover, $\widetilde{u}_{i}$ and $\hat{u}_{i}$ are two filtered velocity fields, obtained through filtering of $u_{i}$ using a low-pass filter with different widths. Thus, in equation (17) $\widetilde{u_{i}}$ and $\widehat{u_{i}}$ are obtained using a different filter width.

In this work we propose a modification for compressible flows, by including the effect of density fluctuations. Thus, we compute the energy ratio as follows

$$
E R=\frac{\sum_{i=1}^{3}\left(\rho_{i} u_{i}-\widetilde{\rho_{i} u_{i}}\right)^{2}}{\sum_{i=1}^{3}\left(\rho_{i} u_{i}-\widehat{\rho_{i} u_{i}}\right)^{2}}
$$

Differently from what is performed in [28, 29, 41] where a top-hat filter is used, in this work we use Moving Least Squares (MLS) based filters. We refer the reader to $[42,43]$ for a complete description of these filters. Here, we only comment that a MLS approximation of a variable can be seen as a low-pass filtering of the variable. This can be written, for a given variable $\Phi$, as

$$
\overline{\Phi_{I}}=\sum_{j=1}^{n} N_{j}(\boldsymbol{x}) \Phi_{j}
$$

where $n$ is the number of neighbors of the stencil of cell $I$, and we use the 
notation $\bar{\Phi}$ to indicate a filtered variable. In equation (19) $\boldsymbol{N}^{T}(\boldsymbol{x})$ are the MLS shape functions, which are computed as exposed in [34]. To compute the MLS shape functions we define an $m$-dimensional basis, which in this case is defined as $\boldsymbol{p}^{T}(\boldsymbol{x})=\left(1, x, y, z, x^{2}, y^{2}, z^{2}, x y, \ldots\right) \in \mathbb{R}^{m}$. Then, the MLS-shape funtions are defined as [34]

$$
\boldsymbol{N}^{T}(\boldsymbol{x})=\boldsymbol{p}^{T}(\boldsymbol{x}) \boldsymbol{M}^{-1}(\boldsymbol{x}) \boldsymbol{P}(\boldsymbol{x}) \boldsymbol{W}(\boldsymbol{x})
$$

where $\boldsymbol{P}=\left[\boldsymbol{p}^{T}\left(\boldsymbol{x}_{\boldsymbol{j}}\right)\right]_{j}$, is a $m \times n_{i}$ matrix where the basis functions are evaluated at each point of the stencil, and $\boldsymbol{M}(\boldsymbol{x})$ is the $m \times m$ moment matrix given by

$$
M(x)=P(x) W(x) P^{T}(x) .
$$

The kernel function $W$ determines the properties of the filter, required in the computation of $\boldsymbol{N}^{T}(\boldsymbol{x})$. We have chosen to use an exponential kernel, defined as [43]

$$
W\left(x, x^{*}, \kappa_{x}\right)=\frac{e^{-\left(\frac{s}{c}\right)^{2}}-e^{-\left(\frac{d m}{c}\right)^{2}}}{1-e^{-\left(\frac{d m}{c}\right)^{2}}}
$$

with $s=\left|x_{j}-x^{*}\right|, d_{m}=\max \left(\left|x_{j}-x^{*}\right|\right)$, with $j=1, \ldots, n_{x^{*}}, c=\frac{d_{m}}{2 \kappa}, x$ is the position of every cell centroid of the stencil and $\kappa$ is a shape parameter.

As stated in equation (18) ER can be seen as a ratio of the spatial highfrequency components of the velocity field for two different filters. Here, when the ER has a value larger than 0.55 , it is considered that there is excessive energy at small scales of the flow, which should be dissipated. When this happens, $\epsilon$ is increased. When ER is smaller than 0.5 the dissipation introduced by the numerical flux is excessive, and $\epsilon$ is reduced. It is important 
to remark that we have chosen different values to define the range of ER than those presented in [28]. The reason is that we have used different filters than those presented in previous works. However, it is important to note that it is possible to get similar results using another configuration of the filters if an adequate ER interval is found, since the range of validity is completely dependent of the filter chosen [44]. Here, we use two different MLS filters to compute ER. One filter with parameters $\kappa=4$ for the computation of $\widetilde{\rho_{i} u_{i}}$ and other with $\kappa=3$ to compute $\widehat{\rho_{i} u_{i}}$. The transfer function of these filters is plotted in figure 1 .

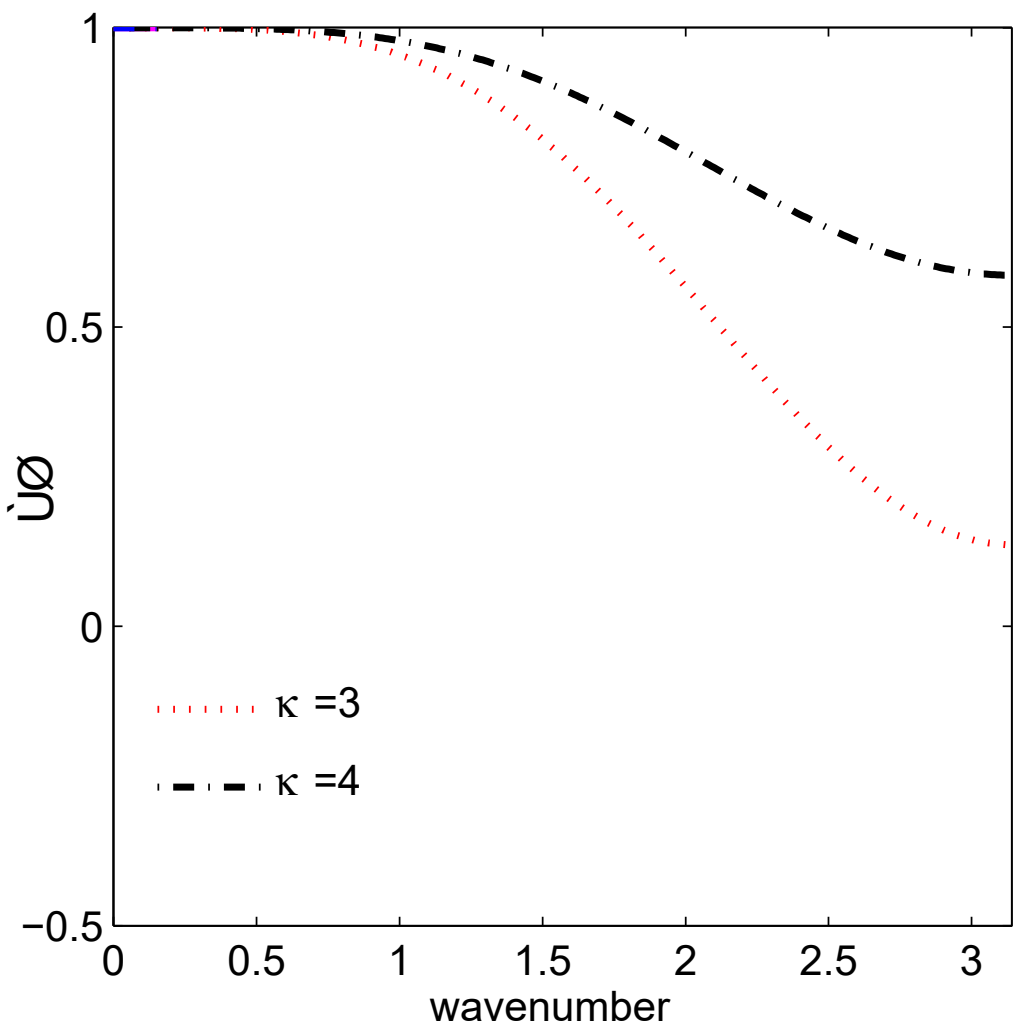

Figure 1: Transfer function $(S F)$ of the MLS filter for different values of $\kappa$. 
In order to automatically adjust the parameter, we follow the rule proposed in [28]

$$
\begin{cases}E R<0.5, & \epsilon=\max [(\epsilon-\phi), 0] \\ E R>0.55, & \epsilon=\min [(\epsilon+\phi), 1] \\ 0.5 \leq E R \leq 0.55, & \epsilon \text { does not change }\end{cases}
$$

Here, a value of $\phi=0.05$ is used, to adjust the value of $\epsilon$ continuously and gradually. In order to keep the conservative character of the numerical scheme, we have to ensure a single value of the $\epsilon$ coefficient at the interface between cells $i$ and $j$. In order to ensure the robustness of the numerical scheme, it is defined as

$$
\epsilon_{i j}=\max \left[\epsilon_{i}, \epsilon_{j}\right]
$$

It is important to remark that, when applied to non-smooth flows, this method alone may lead to spurious oscillations and eventually to the crash of computations, since the reduced viscosity may not be enough to stabilize the computations near shocks. In order to extend the applicability of this method to turbulent compressible flows, we propose to combine the ADA method with the a posteriori paradigm [30,31]. This will be addressed in the next section.

\subsection{The a posteriori paradigm}

The use of the ADA method in the framework of compressible flow is problematic, since the algorithm of equation (23) may not be fast enough to introduce dissipation in the presence of a shock wave. Moreover, in the 
context of high-order methods, even a value of $\epsilon=1$ could be not enough for stabilization since high-order schemes are not monotonic. Thus, a method-

ology for stabilizing the computations is required if shocks appear in the solution, but with the minimum interference to the ADA method. In this work we adapt the a posteriori paradigm [30,31] to the computation of compressible turbulent flows with the ADA method.

The main idea of this approach is to compute, each step of time integration algorithm, the cell averaged values using the most accurate available scheme. This solution is called candidate solution. Then, we use a chain of different criteria to evaluate if the candidate solution is admissible or not. In this context, admissible means that it gives positive densities and pressures, and that the level of unphysical oscillations is low. In the original version of this methodology [30] an iterative scheme was proposed for reducing progressively and locally the order of the numerical scheme, recomputing the solution and evaluating again if the solution is admissible. This evaluation/order reduction procedure is performed until the solution is considered admissible or the numerical scheme reaches first-order, which always gives admissible solutions. This procedure is called Multidimensional Optimal Order Detection (MOOD) [30, 31]. In the following we expose the adapted procedure proposed in this work.

\subsubsection{A posteriori detection}

Once the candidate solution is computed, the following chain of detectors is used

Physical Admissible Detector (PAD)[30]: This detector checks if 
the candidate solution has positive density and positive pressure. Thus, if the candidate solution has negative values of pressure and/or density in a cell, or even a NaN value, this cell is marked as not good and is recomputed again using a lower order scheme.

Numerical Admissible Detector (NAD) [45]: It is a relaxed version of the Discrete Maximum Principle (DMP)[30]. It checks if the solution is monotonic and new local extrema is not created.

$$
\min _{\mathbf{y} \in \mathcal{V}_{i}}\left(\mathbf{U}^{n}(\mathbf{y})\right)-\delta \leqslant \mathbf{U}^{*}(\mathbf{x}) \leqslant \max _{\mathbf{y} \in \mathcal{V}_{i}}\left(\mathbf{U}^{n}(\mathbf{y})\right)+\delta
$$

In equation (25) superscript $n$ indicates the previous Runge-Kutta step, and $\mathbf{U}^{*}(\mathbf{x})$ is the candidate solution. The $\delta$ parameter allows a certain level of tolerance, and it is defined as in [45]

$$
\delta=\max \left(10^{-4}, 10^{-3} \cdot\left(\max _{\mathbf{y} \in \mathcal{V}_{i}}\left(\mathbf{U}^{n}(\mathbf{y})\right)-\min _{\mathbf{y} \in \mathcal{V}_{i}}\left(\mathbf{U}^{n}(\mathbf{y})\right)\right)\right)
$$

Equation (25) expresses the fact that the representation of the candidate solution in a cell must remain between the minimum and the maximum values of the solution at the previous time step in the considered set. The small number delta in (25) is a parameter used to relax the discrete maximum principle. It is defined in (26), in a way to allow the candidate solution to exceed the extrema only by a small fraction of the total jump of the variable considered.

Thus, in order to keep a high accuracy when dealing with smooth extrema, very small undershoots and overshoots are allowed. If the condition expressed in equation (25) is verified, the solution is considered as admissible. 
Note that in order to work with this formulation, all the variables in equation (26) should be normalized with an adequate reference value to get a value between 0 and 1 . In the original formulation $[30,31,45]$ the set $\mathcal{V}_{i}$ represents the set of first neighbors of the point $\mathrm{x}$. However, in this work $\mathcal{V}_{i}$ is defined as the stencil used by MLS approximations [34, 35, 36]. This is based on the ideas presented in [3] in the context of slope limiters. The fullfillment of the NAD condition implies that the candidate value remains between the local minimum and the local maximum of the previous time step. In all of the examples of this work, the NAD is checked only in one variable (density or energy), but it could be applied to the full vector of conservative variables as suggested by [31].

If one cell does not verify the PAD criteria, it is marked and recomputed with a first-order scheme and $\epsilon$ is set to $\epsilon=1$. If the cell does not verify the NAD criteria, the cell is recomputed using a first-order scheme. For these cells, the use of $\epsilon=1$, even though is a possible choice, will lead to an excessive dissipation. So we have to define a more accurate value for $\epsilon$. One possibility is to keep the value of $\epsilon$ given by the ADA method. However, if there are strong shocks in the problem, the stability of the numerical scheme is not guaranteed. In order to solve this problem, the value of $\epsilon$ is chosen according to the strength of the shock detected. In order to determine the strength of the shock, we compute the shock strength parameter $(F)$ proposed in [46], defined in terms of the pressure ratio $y=\frac{p_{L}}{p_{R}}$ as

$$
F=\frac{1}{4}\left(y+2+\frac{1}{y}\right)
$$

An alternative is to use the density ratio $s=\frac{\rho_{L}}{\rho_{R}}$ instead of the pressure 
ratio. In this case, $F$ is defined as [46]

$$
F=\frac{\frac{1}{2} \gamma^{2} /\left(\gamma^{2}-1\right)-\frac{1}{2}}{N-\frac{1}{2}}
$$

where $N$ is

$$
N=\frac{(1+\mu)^{2}}{2 \mu} \frac{s}{(1+s)^{2}}
$$

with $\mu=\frac{\gamma+1}{\gamma-1}$.

Using these definitions, the parameter $F$ varies in $[1, \infty)$. Once the strength of the shock is determined using the $F$ parameter, a value of $\epsilon_{\text {shock }}$ for this cell is computed as follows.

We define the value of $\epsilon$ to vary following an hyperbolic tangent as

$$
\epsilon_{\text {shock }}=\frac{1}{2}\left(1+\tanh \left(\frac{F-F_{0}}{l_{0}}\right)\right)
$$

In this work, the values of $F_{0}=1.01$ and $l_{0}=0.0085$ are used. A plot of this curve is shown in figure 2. It is observed the fast increase of the value of epsilon which ensures stability for the computations. Note that the choice of these parameters determines the minimum value of $\epsilon$ and also the strength of the shock for which the scheme recovers the full dissipation. All the numerical examples of this work have been computed using these values of $F_{0}$ and $l_{0}$.

To summarize, for a given cell $i$ where the NAD is activated, we define

$$
\epsilon_{N A D}=\max \left(\epsilon_{\text {shock }}, \epsilon_{A D A}\right)
$$

where $\epsilon_{A D A}$ refers to the value given by the ADA algorithm as explained in previous sections.

The complete algorithm is schematically shown in figure 3 . 


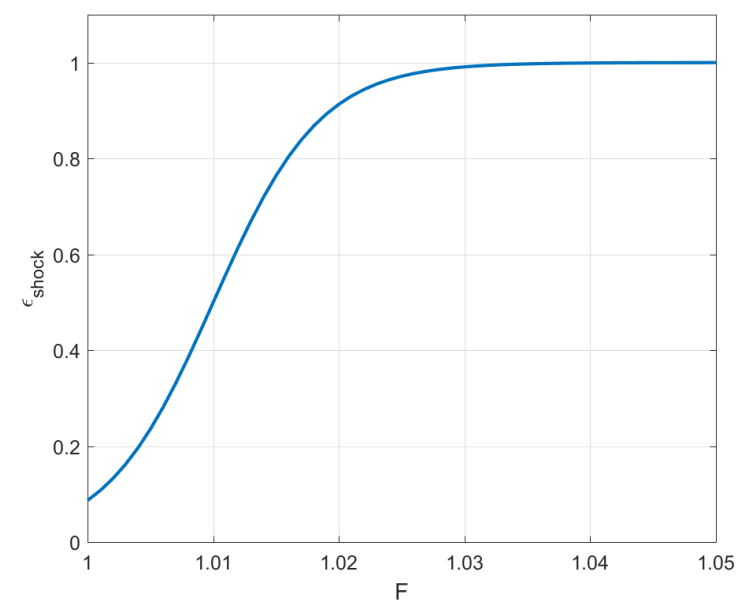

Figure 2: Value of $\epsilon_{\text {shock }}$ in terms of the shock strength parameter $F$ [46]

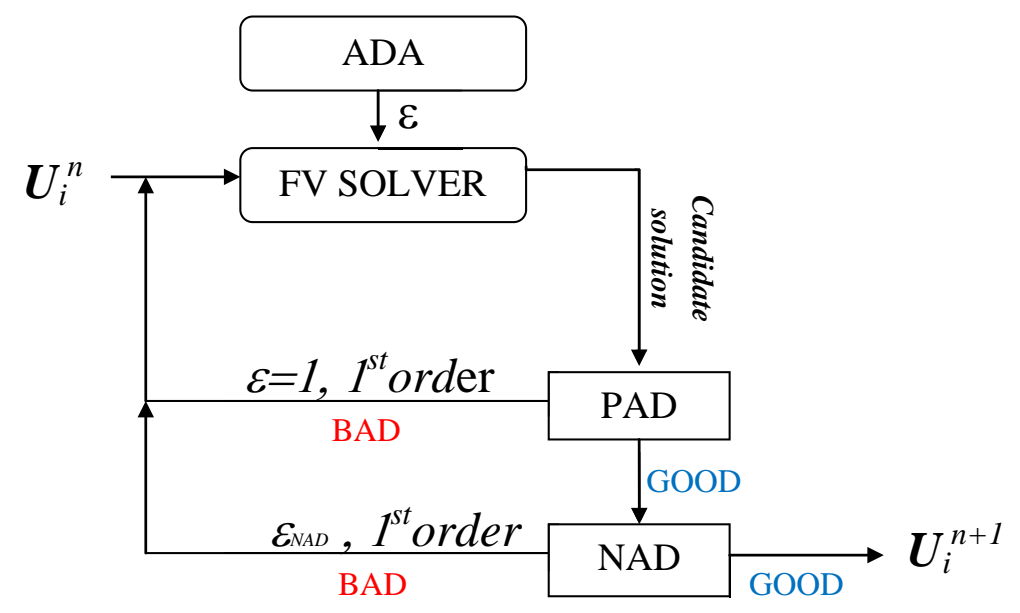

Figure 3: Schematic representation of the proposed algorithm.

Note that in the practical application of this methodology, only the detected cells have to be recomputed $[30,31]$ once the candidate solution has been checked. 


\section{Numerical tests}

In this section we test the proposed approach for the resolution of underresolved turbulent flows in an Implicit LES approach. First we address two $1 \mathrm{D}$ problems in order to show that the modification of the dissipation does not introduce dispersion errors. All the examples are solved using a thirdorder FV-MLS method [13, 14]. In all of the numerical examples presented here we have used a third-order TVD Runge-Kutta scheme [47] for time integration.

\subsection{One-dimensional tests}

In this section we test the proposed methodology in two one-dimensional tests, in order to study if the proposed approach is stable. First we test the one-dimensional advection equation, and then, the 1D Euler equations are solved for several test cases. These test cases with Euler equations are intended to test the robustness of the proposed scheme when dealing with discontinuities. Note that due to the configuration of these tests cases for the Euler equation, it is not expected to obtain a remarkable improvement in accuracy with the adaptive viscosity method. This is due to the fact that when a strong shock is detected, the scheme quickly recovers the full

dissipation whereas in the rest of the domain, the dissipation introduced by the original scheme is low.

\subsubsection{Linear Advection equation}

In this first case we test if the reduced dissipation introduce dispersion errors in the solution. The computational domain is $\Omega=[0,4]$, with periodic 
boundary conditions. We initialize the computations using the exact solution, which at a given time $t$ reads

$$
u(x, t)=1+A \sin (\pi(x-a t))
$$

where $A=0.2$ and the constant freestream velocity $a=1$. The simulation is run until $t=4$, using the Lax-Friedrichs flux with and without the ADA method presented in section 3.1. The results are shown in figure 4 and Table 1 where $L_{2}$-norm of the error and the convergence order are computed and presented.

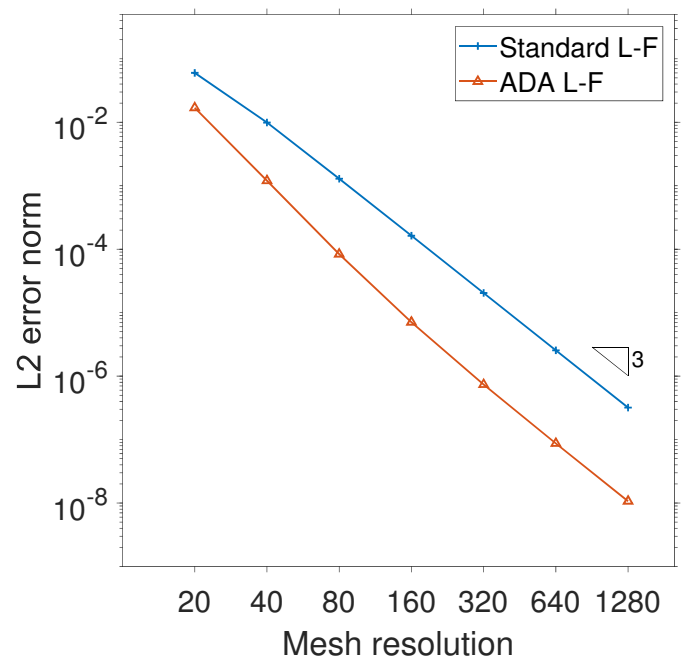

Figure 4: One-dimensional linear advection test case. Comparison of $L_{2}$ error norms with and without the proposed method at $t=4$.

It is observed that the ADA method recovers the expected order of convergence, with increased accuracy compared with the baseline method. 


\begin{tabular}{ccccc}
\hline & \multicolumn{2}{c}{ FV-MLS } & \multicolumn{2}{c}{ FV-MLS ADA } \\
\hline Grid & $L_{2}$ error & order & $L_{2}$ error & order \\
\hline 20 & $6.00 \mathrm{E}-02$ & - & $1.69 \mathrm{E}-02$ & - \\
\hline 40 & $9.89 \mathrm{E}-03$ & 2.60 & $1.20 \mathrm{E}-03$ & 3.82 \\
\hline 80 & $1.29 \mathrm{E}-03$ & 2.93 & $8.36 \mathrm{E}-05$ & 3.85 \\
\hline 160 & $1.63 \mathrm{E}-04$ & 2.99 & $7.07 \mathrm{E}-06$ & 3.56 \\
\hline 320 & $2.04 \mathrm{E}-05$ & 3.00 & $7.37 \mathrm{E}-07$ & 3.26 \\
\hline 640 & $2.55 \mathrm{E}-06$ & 3.00 & $8.69 \mathrm{E}-08$ & 3.08 \\
\hline 1280 & $3.19 \mathrm{E}-07$ & 3.00 & $1.07 \mathrm{E}-08$ & 3.02 \\
\hline \hline
\end{tabular}

Table 1: $L_{2}$-norm of the error and convergence order for the linear advection equation test case, using the proposed and the baseline methods.

\subsubsection{Isolated steady normal shock}

In this case we compute a stationary shock. The computational domain is $[0,20]$, and the shock is placed at $x_{0}=10$. We solve this problem using 100 control volumes until an steady state is reached, using the Rusanov numerical flux. The left initial state is defined as $\left(\rho_{L}, u_{L}, p_{L}\right)=\left(1,1, \frac{1}{\gamma M_{L}^{2}}\right)$ and the right initial state is computed using with the following expressions, obtained from the Rankine-Hugoniot conditions [40]

$$
\begin{aligned}
\rho_{R} & =\frac{(\gamma+1) M_{L}^{2}}{(\gamma-1) M_{L}^{2}+2} \\
u_{R} & =\frac{1}{\rho_{R}} \\
p_{R} & =\left[1+\frac{2 \gamma}{\gamma+1}\left(M_{L}^{2}-1\right)\right] \frac{1}{\gamma M_{L}^{2}}
\end{aligned}
$$


where the Mach number of the left state is $M_{L}=7$ and the polytropic index is $\gamma=1.4$.
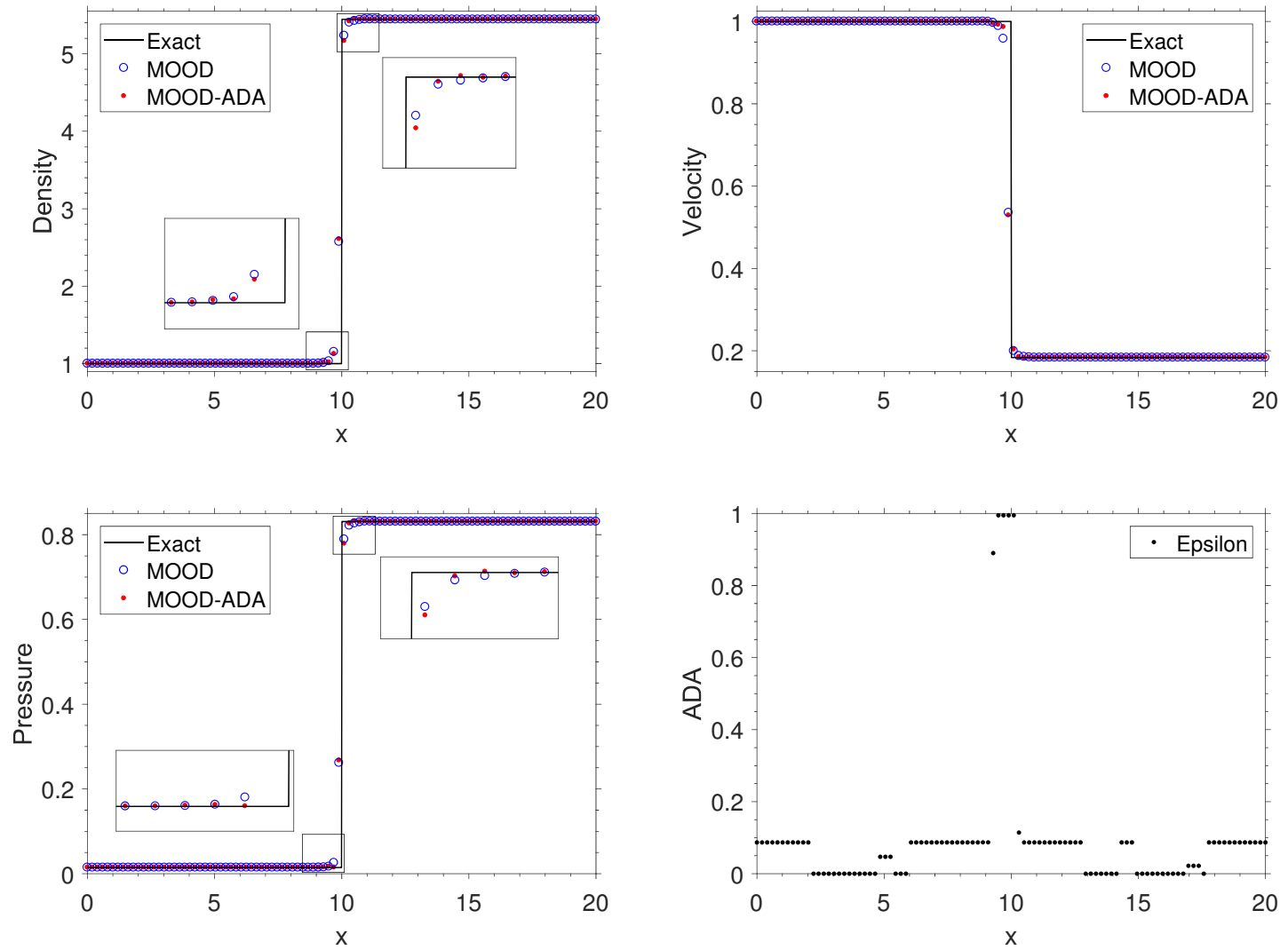

Figure 5: Isolated stationary shock test case. Comparison of the results using adaptive dissipation and the Rusanov numerical flux..

The results are shown in figure 5. It is shown that both, MOOD and ADA-MOOD scheme are able to obtain a solution free of oscillations. The adaptive dissipation scheme obtains a slightly sharper shock front. It is observed that the value of $\epsilon$ (and then the dissipation) is one at the shock and is very reduced in the rest of the domain. 


\subsubsection{Slowly moving shock}

This case computes a strong slowly moving shock. Note that the lower value of the pressure in the right state makes the pressure ratio close to its maximum. Thus, it is an almost infinite strong shock. This is a severe test case for the numerical scheme, and we reproduce here the configuration given in [48]. The computational domain is $[0,100]$, and the shock is initially placed at $x_{0}=20$. We solve this problem using 100 control volumes until a final time of $t=2000$ with $\mathrm{CFL}=0.5$ using the Rusanov numerical flux.

The left and right initial states are defined as in $[48]\left(\rho_{L}, u_{L}, p_{L}\right)=(4,0.3,4 / 3)$ and $\left(\rho_{R}, u_{R}, p_{R}\right)=\left(1,-1.3,10^{-6}\right)$. The polytropic index is $\gamma=5 / 3$. The results are shown in figure 6. It is shown that both, MOOD and ADA-MOOD schemes are able to obtain a solution free of oscillations. Moreover, the shock position is well-predicted. As in the previous case, it is observed that the value of $\epsilon$ (and then the dissipation) is one at the surroundings of the shock and it is very reduced in the rest of the domain.

\subsubsection{Isolated contact discontinuity}

In this test we show the behavior of the proposed methodology when dealing with contact discontinuities. In particular, we test both, an isolated stationary contact discontinuity and also an isolated moving contact discontinuity. The left and right initial states are defined as $\left(\rho_{L}, u_{L}, p_{L}\right)=(1,0,0.5)$ and $\left(\rho_{R}, u_{R}, p_{R}\right)=(0.6,0,0.5)$ for the stationary case. The contact discontinuity is placed at $x_{0}=0.5$. The polytropic index is $\gamma=1.4$. We discretize the domain $[0,1]$ using 100 control volumes. The moving case uses the same initial states with an advection velocity $u=0.1$. We use also 100 control volumes and the computational domain is enlarged to $[0,5]$, and the contact 

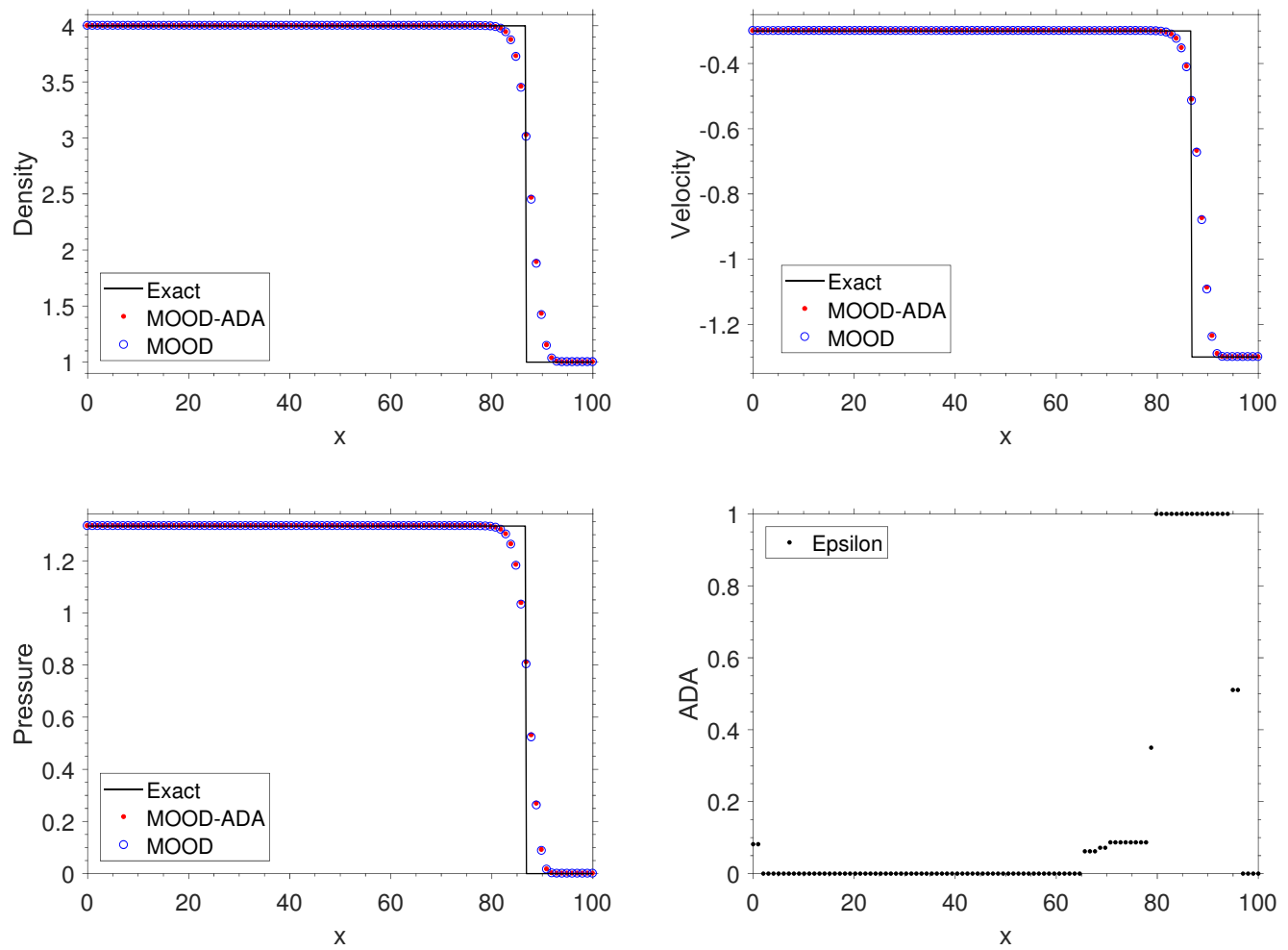

Figure 6: Slowly moving shock test case. Comparison of the results using adaptive dissipation and the Rusanov numerical flux at $t=2000$.

discontinuity is initially located at $x_{0}=2$. Rusanov flux is used for the computations. And for this test case we use the expression of $F$ given in equation (28).

The results are displayed in figure 7 . It is observed the reduced numerical viscosity obtained with the MOOD-ADA method. In the case of the stationary contact discontinuity, the solution is excellent, with only 2 cells in the discontinuity. In the moving case, the numerical viscosity is also reduced compared with the full Rusanov flux. 

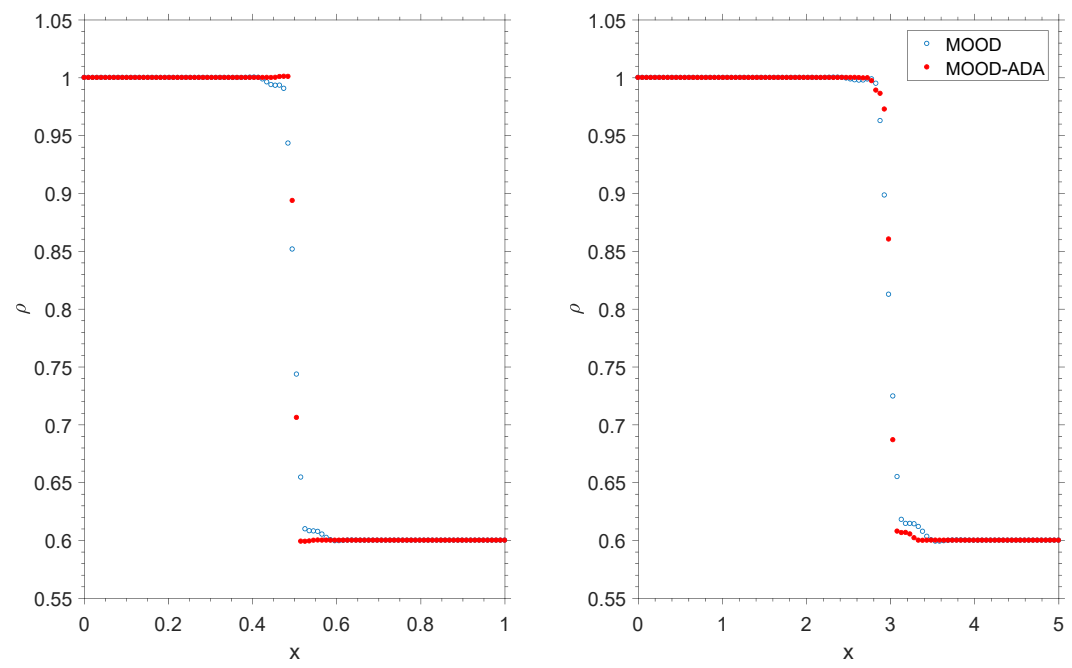

Figure 7: Isolated contact discontinuity. Comparison of the results for the density field using the baseline and adaptive dissipation methods. On the left, results of the stationary case at $t=1$. On the right, results for the moving case at $t=10$.

\subsubsection{Double shock}

In this test case, a left and right shocks collide, and the solution consists of a left facing shock that travels very slowly to the right, a contact discontinuity which travels to the right and a right traveling shock wave. The two different states are initially placed in a $[0,1]$ domain, separated at $x_{0}=0.5$. The left and right initial states are defined as $\left(\rho_{L}, u_{L}, p_{L}\right)=(6,20,450)$ and $\left(\rho_{R}, u_{R}, p_{R}\right)=(6,-6,45)$. We solve this problem using 100 control volumes until a final time of $t=0.02$ and the numerical flux of Roe.

The results using the proposed methodology are compared with the case using a constant value of $\epsilon=1$. The exact solutions were computed using the NUMERICA software [49]. The results are shown in figure 8. We observe that the use of adaptive dissipation does not vary the results. This is an 
expected results for this test case, since in the zone of the shock we recover the original scheme, whereas in the rest of the domain, the dissipation introduced by the original scheme is low.
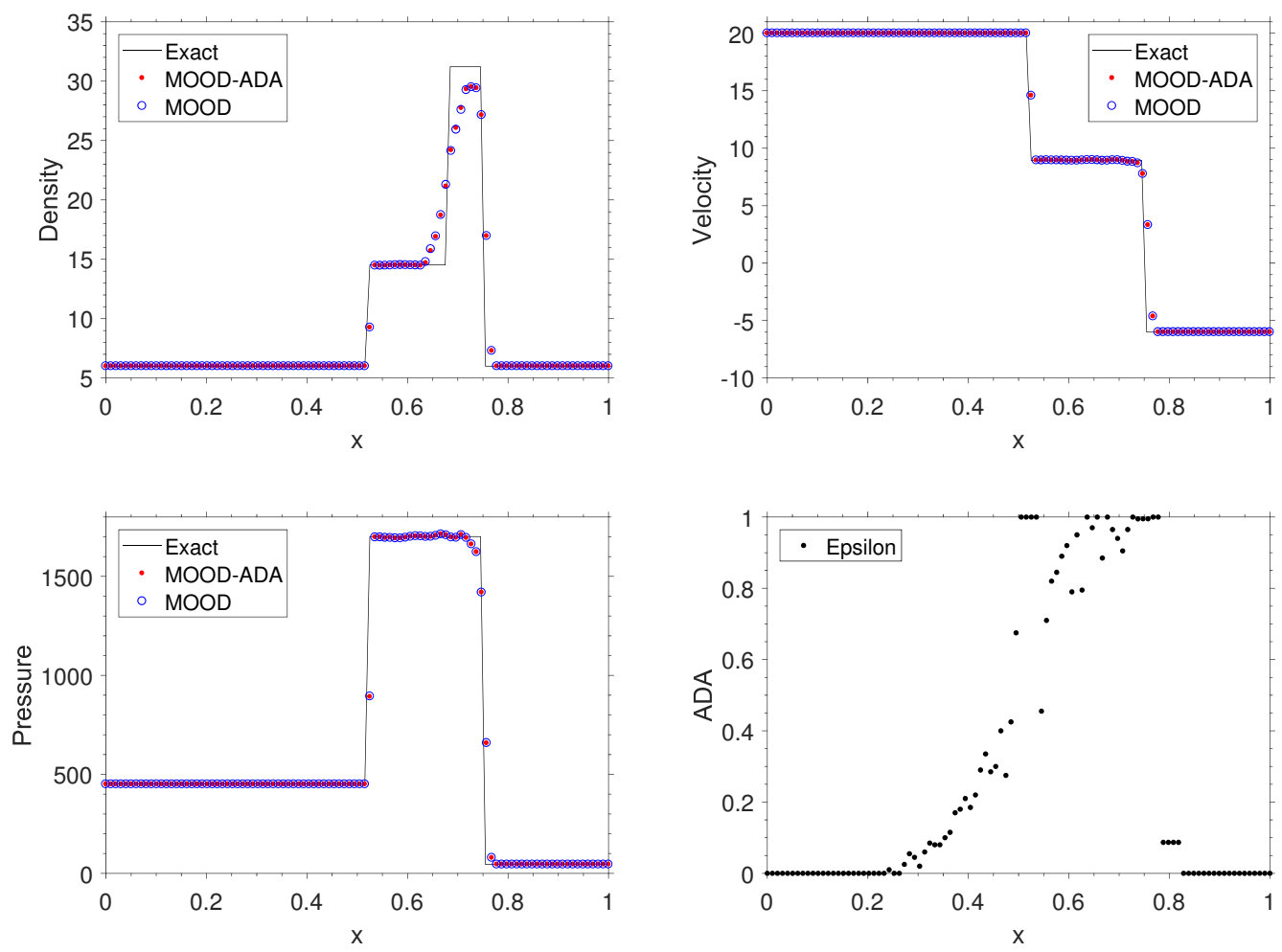

Figure 8: One-dimensional double shock tube test case. Comparison of the results using adaptive dissipation and the numerical flux of Roe at $t=0.02$.

\subsection{Isentropic vortex convection}

This validation case corresponds to the unsteady vortex convection. This test case is widely used as benchmark for unsteady vortical flows [50, 51, 52]. 
The analytical solution reads as

$$
\begin{aligned}
\frac{u(x, y, t)}{a_{\infty}} & =\frac{u_{\infty}}{a_{\infty}}-\frac{K}{2 \pi a_{\infty}} \hat{y} e^{\alpha\left(1-r^{2}\right) / 2} \\
\frac{v(x, y, t)}{a_{\infty}} & =\frac{v_{\infty}}{a_{\infty}}+\frac{K}{2 \pi a_{\infty}} \hat{x} e^{\alpha\left(1-r^{2}\right) / 2} \\
\frac{T(x, y, t)}{T_{\infty}} & =1-\frac{K^{2}(\gamma-1)}{8 \alpha \pi^{2} a_{\infty}^{2}} e^{\alpha\left(1-r^{2}\right)} \\
\frac{\rho(x, y, t)}{\rho_{\infty}} & =\left(\frac{T(x, y, t)}{T_{\infty}}\right)^{\frac{1}{\gamma-1}} \\
\frac{p(x, y, t)}{p_{\infty}} & =\left(\frac{T(x, y, t)}{T_{\infty}}\right)^{\frac{\gamma}{\gamma-1}}
\end{aligned}
$$

where $\hat{x}=x-x_{0}-u_{\infty} t, \hat{y}=y-y_{0}-v_{\infty} t, \gamma=1.4$ and $r=\sqrt{\hat{x}^{2}+\hat{y}^{2}}$. Here, the chosen parameters are $\alpha=1, \rho_{\infty}=1, p_{\infty}=1,\left(u_{\infty}, v_{\infty}\right)=\left(\frac{0.5}{\sqrt{2}}, \frac{0.5}{\sqrt{2}}\right)$, $\left(x_{0}, y_{0}\right)=(-5,-5)$ and $K=5$. This corresponds to a free stream Mach number of $M=0.5$. With this set of parameters the vortex starts at the position $(x, y)=(-5,-5)$ and at reaches the position $(x, y)=(5,5)$ at $t=20 \sqrt{2}$.

The proposed methodology is used to compute the problem and compared with the results obtained without the adaptive dissipation method. Error norms and convergence order are reported in Table 2. In this case, the ADA scheme obtains the same results using Rusanov and Roe fluxes. The reason is that, for this case, the ADA method leads to the central scheme, neglecting the dissipation introduced by the numerical fluxes. Note that this result is not expected to hold for more complex flows. 


\begin{tabular}{cccccccccc}
\hline \multicolumn{9}{c}{ Rusanov } & \multicolumn{3}{c}{ Roe } \\
\hline \multicolumn{1}{c}{ Standard } & \multicolumn{2}{c}{ ADA } & & Standard & \multicolumn{2}{c}{ ADA } \\
\hline Grid & $L_{2}$ error & order & $L_{2}$ error & order & $L_{2}$ error & order & $L_{2}$ error & order \\
\hline $32 \times 32$ & $6.00 \mathrm{E}-02$ & - & $5.27 \mathrm{E}-02$ & - & $5.10 \mathrm{E}-02$ & - & $5.28 \mathrm{E}-02$ & - \\
\hline $64 \times 64$ & $3.04 \mathrm{E}-02$ & 0.98 & $3.90 \mathrm{E}-03$ & 3.75 & $1.84 \mathrm{E}-02$ & 1.47 & $3.90 \mathrm{E}-03$ & 3.76 \\
\hline $128 \times 128$ & $7.74 \mathrm{E}-03$ & 1.98 & $2.51 \mathrm{E}-04$ & 3.96 & $4.37 \mathrm{E}-03$ & 2.07 & $2.51 \mathrm{E}-04$ & 3.96 \\
\hline $256 \times 256$ & $1.75 \mathrm{E}-03$ & 2.14 & $2.01 \mathrm{E}-05$ & 3.64 & $7.85 \mathrm{E}-04$ & 2.48 & $2.01 \mathrm{E}-05$ & 3.64 \\
\hline $512 \times 512$ & $2.57 \mathrm{E}-04$ & 2.77 & $3.20 \mathrm{E}-06$ & 2.65 & $1.06 \mathrm{E}-04$ & 2.89 & $3.20 \mathrm{E}-06$ & 2.65 \\
\hline
\end{tabular}

Table 2: $L_{2}$-norm of the error and convergence order for the isentropic vortex convection test case using Rusanov and Roe fluxes.

\subsection{Incompressible isotropic Taylor-Green vortex}

The Taylor-Green vortex (TGV) is the simplest model for the analysis of the nonlinear transfer of kinetic energy among the different scales of a flow. Even if it is simple to construct, it contains several key physical processes of turbulence. We solve the inviscid version of this test example in order to analyze an infinite Reynolds case. This is intended to show the behavior of the proposed method in under-resolved simulations, and to examine the capability of the ILES scheme to reproduce transition to turbulence. For very large Reynolds number, it is known that statistically isotropic turbulence develops following the $-5 / 3$ decay Kolmogorov's law of the kinetic-energy spectra within the inertial subrange around $t \approx 9$ [53]. A physically-consistent numerical method developed for implicit LES should recover this behavior. 


\subsubsection{Setup of the problem and results}

In this test case, we solve the three-dimensional Euler equations with $\gamma=5 / 3$, using both, Roe and Rusanov numerical fluxes. The initial condition of the TGV is

$$
\begin{aligned}
& u(x, y, z, 0)=\sin (x) \cos (y) \cos (z) \\
& v(x, y, z, 0)=-\cos (x) \sin (y) \cos (z) \\
& w(x, y, z, 0)=0 \\
& \rho(x, y, z, 0)=1 \\
& p(x, y, z, 0)=100+\frac{1}{16}[(\cos (2 x)+\cos (2 y))(2+\cos (2 z))-2]
\end{aligned}
$$

We solve this set of equations in a periodic $[0,2 \pi] \times[0,2 \pi] \times[0,2 \pi]$ cube, using a $64^{3}$ grid, until a final time of $t=10$. This grid is used in order to check the behavior of the method for a under-resolved turbulent simulation. Since the density must remain constant in this case, we use the kinetic energy as the variable to detect oscillations with the a posteriori method (equation (25)). The evolution of the normalized total kinetic energy and enstrophy using the proposed scheme with Roe and Rusanov numerical fluxes, is shown in figure 9, where our results are also compared with those obtained using other numerical schemes. In particular, we compare with the eight-order TENO scheme [33], the Fourier collocation method with exponential filter (F-EF-10-38-N) and the WENO5 scheme [54]. We also compare with the results obtained with a third-order Residual Based Compact scheme (RBC3) [55]. The non linear interactions generate successively smaller scales, but the kinetic energy remains constant until $t \approx 4$. The results obtained by the proposed approach, are in excellent agreement with the reference solution, both 
in terms of the kinetic energy and enstrophy. The use of the ADA method allows to obtain comparable or better results than those attained with higher order schemes. The method also reproduces closely the semi-analytical results presented in [56]. It is also seen that the results obtained with the third-order FV-MLS method with a constant value of $\epsilon=1$ (that is, without the ADA method) seem to be less dissipative than those obtained with the proposed methodology. These unexpected results are explained since in the scheme with the ADA method, the reduced dissipation introduced from the reduction of $\epsilon$ leads to more frequent activation of the a posteriori stabilization method. This, which at a first sight could seem a drawback of the proposed method, turns in fact a numerical mechanism to capture the right dynamics of the flow. The ADA method adjusts the dissipative part of the numerical flux regarding the high-frequency content of the solution, increasing the dissipation where the solution is under-resolved, and decreasing the dissipation where the high-frequency content of the solution is low. However, there are sudden events (such as a shock wave, or a sudden collapse of a vortex in high-Reynolds flows (with practically no viscosity)) where the ADA method is not able to react instantaneously, since the variation of epsilon is limited each time step according to equation (23). In these scenarios, the MOOD algorithm increases the dissipation. Moreover, there is one possible scenario in which the MOOD plays an unexpected role: when the solution is locally under-resolved, the ADA algorithm may increase the value of $\epsilon$ until a magnitude of $\epsilon=1$ without triggering the MOOD. In this case, it is even possible that the numerical viscosity introduced by the high-order scheme would not be enough to dissipate the content of energy required to follow 
Kolmogorov's law. In this case, the high-frequency content of the solution will increase until a level where the MOOD is triggered. This shows that very high-order methods (with very low intrinsic dissipation) could face accuracy problems for ILES of very under-resolved flows without a numerical mechanism to introduce a higher amount of viscosity such as the one proposed in this work.

In this test case, the dissipation introduced by the third-order scheme is low, but enough to dissipate the oscillations (avoiding the activation of the a posteriori stabilization method). However, it still introduce an excessive amount of numerical dissipation that avoids the scheme to accurately reproduce the physics of the flow. This is confirmed in figure 10 (left), where the evolution of the kinetic energy spectrum is plotted for several times. It is observed that for time $t=10$ the proposed numerical method with the numerical flux of Roe is able to reproduce the Kolmogorov scaling, whereas the spectrum obtained using a constant value of $\epsilon=1$ does not capture the physical behavior. Moreover, in figure 10 (right), it is shown that the kinetic energy decays as $t^{-1.3}$. This value is in the range 1.2-1.4 which is in good agreement with the values obtained in the literature for the isotropic decay of turbulence $[33,57]$.

In figure 11 (left) we plot the energy spectrum obtained using the Rusanov flux. Note that the use of the proposed methodology allows us to improve the results compared to those obtained with the original scheme. Even though the results obtained with Rusanov flux are not as accurate as those using the Roe flux, the improvement in accuracy is quite remarkable. This is an important result for cases where Roe's flux is not applicable. In figure 11 

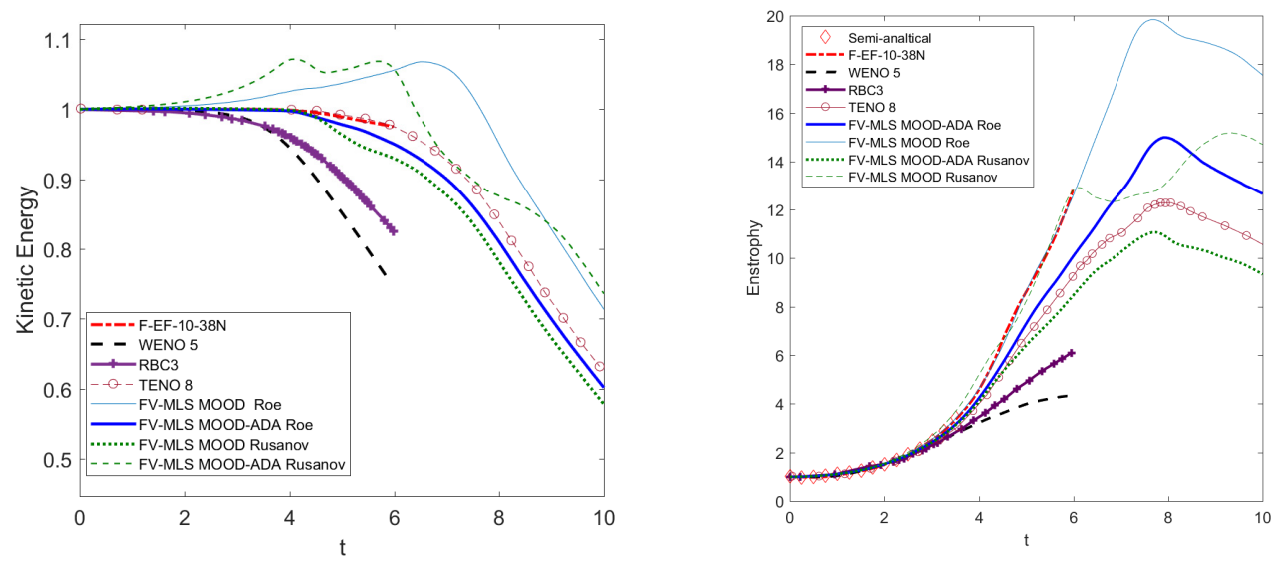

Figure 9: Incompressible isotropic Taylor-Green vortex. Evolution of the normalized total kinetic energy (left) and enstrophy (right), obtained with the proposed scheme (FV-MLS MOOD-ADA) using a $64^{3}$. The results for the TENO-8 [33], the third-order Residual Based Compact scheme (RBC3) [55], the Fourier collocation method with exponential filter (F-EF-10-38-N) schemes and the WENO5 scheme [54] have been digitized from the indicated references. The results obtained using FV-MLS MOOD third-order schemes without the proposed adaptive viscosity method are also shown. The semi-analytical enstrophy solution of [56] is plotted with diamonds.

(right), it is seen that the kinetic energy decay lies also in the 1.2-1.4 range.

We note that the proposed methodology allows to obtain physical results for under resolved simulations using relatively low order-schemes. The reduced dissipation obtained through the use of the ADA methodology and the a posteriori stabilization to keep the stability, is comparable to that introduced by a very-high order method.

In figure 12 it is shown that in most of the domain the value of $\epsilon$ is less than one. Around $t=5$, the grid is no longer finer enough to solve all the scales and the solution becomes under-resolved. In this moment, the $a$ 

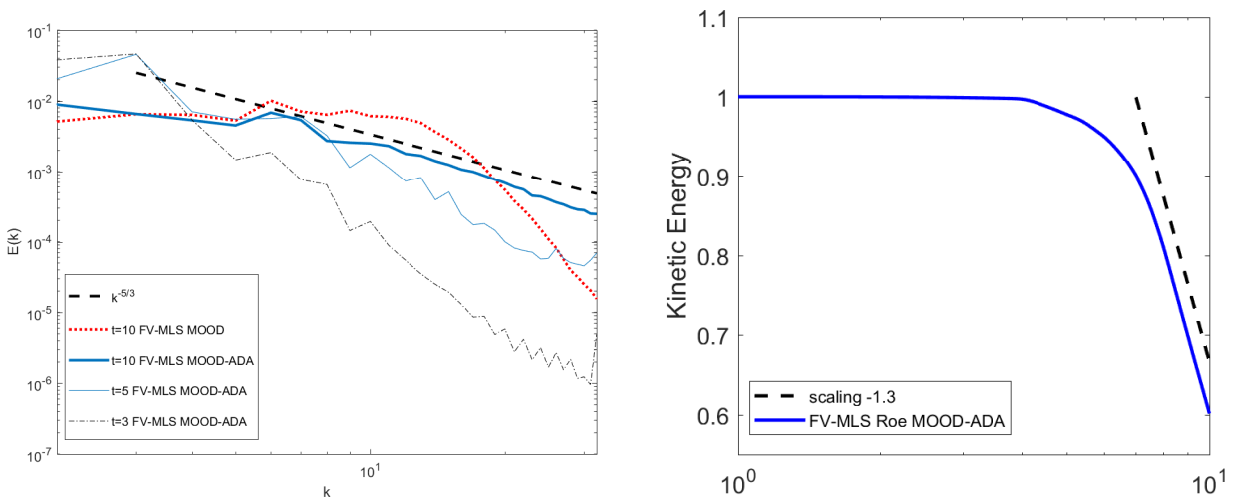

Figure 10: Incompressible isotropic Taylor-Green vortex. The 3D energy spectrum at different times compared with the Kolmogorov scaling is shown on the left. On the right, the evolution of the normalized total kinetic energy with the expected -1.3 scaling is shown. Results obtained using the third-oder FV-MLS MOOD-ADA scheme and the numerical flux of Roe.
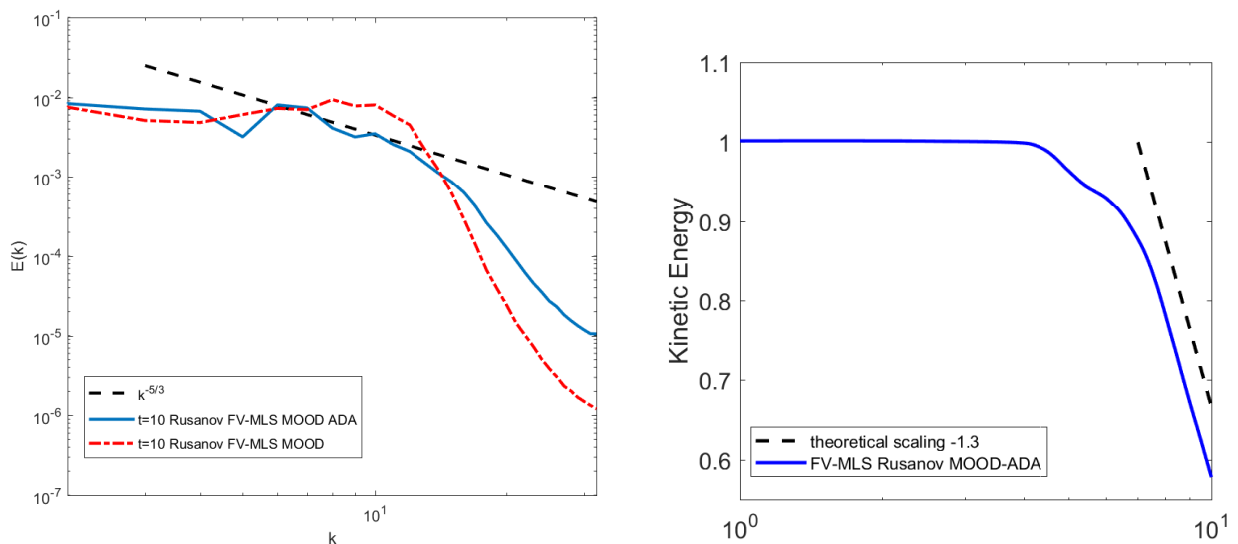

Figure 11: Incompressible isotropic Taylor-Green vortex. The 3D energy spectrum at $t=10$ compared with the Kolmogorov scaling is shown on the left. On the right, the evolution of the normalized total kinetic energy with the expected -1.3 scaling is shown. Results obtained using the third-oder FV-MLS MOOD-ADA scheme and the numerical flux of Rusanov. 
posteriori method is activated in some cells, as it is shown in figure 13. This is related with the collapse of some vortical structures. The activation of the a posteriori method introduce enough viscosity to keep the stability, by decreasing the order of the scheme at the points where the vortical structures collapse.
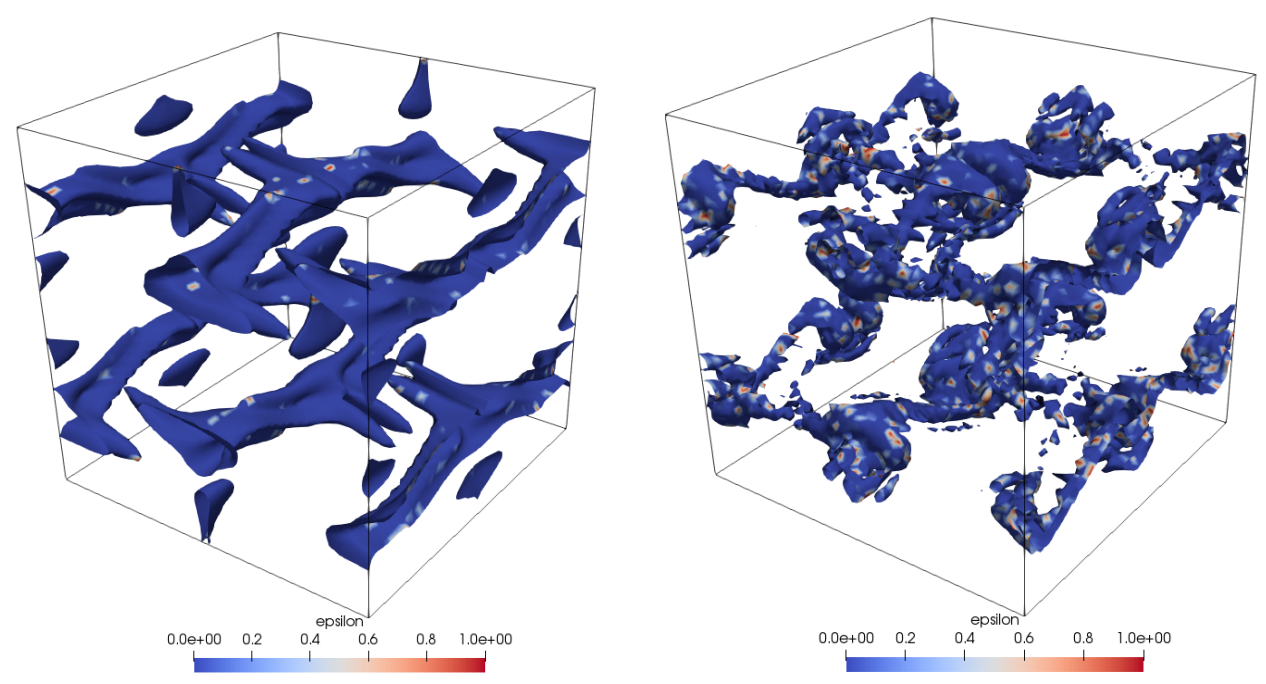

Figure 12: Incompressible isotropic Taylor-Green vortex. Absolute value of the vorticity contours colored with the value of $\epsilon$. On the left, absolute value of the vorticity contours at $t=3$ are shown, and on the right the absolute value of the vorticity contours with a value equal to 0.4 at $t=5$ are plotted. Results obtained using the third-oder FV-MLS MOOD-ADA scheme and the numerical flux of Roe.

\subsection{Decay of Compressible Isotropic Turbulence}

In this section we test the proposed method using the decay of compressible turbulence test case. This test case is a simple case of turbulent flow, but it allows to check the ability of the method for Large Eddy Simulations. It has been used by many authors to investigate SGS models and to test numerical 

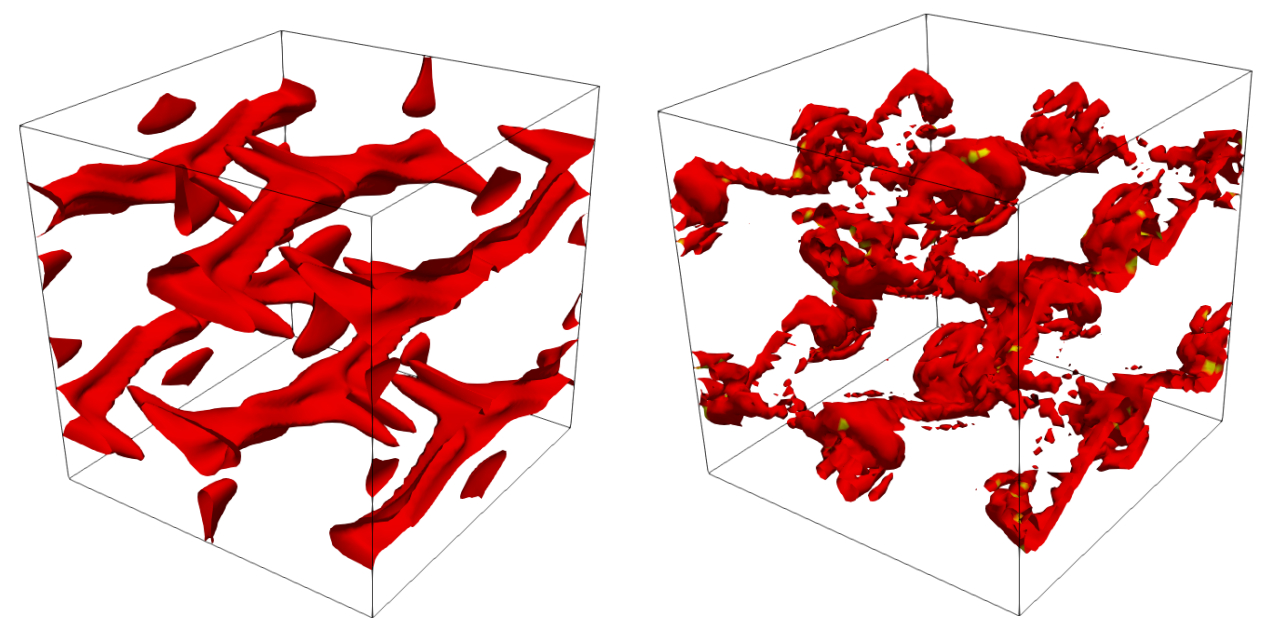

Figure 13: Incompressible isotropic Taylor-Green vortex. Absolute value of the vorticity contours colored with the value of the order of the scheme. On the left, absolute value of the vorticity contours at $t=3$ are shown, and on the right the absolute value of the vorticity contours with a value equal to 0.4 at $t=5$ are plotted. Red color indicates the third-order scheme whereas the yellow color indicates the use of the first-order scheme. Results obtained using the third-oder FV-MLS MOOD-ADA scheme and the numerical flux of Roe.

methods [18, 21, 58, 59, 60, 61]. Roe flux is used in the computations.

\subsubsection{Setup of the problem}

For this problem we solve the three dimensional Navier-Stokes equations with $\gamma=1$.4. We consider a computational domain that is a $2 \pi \times 2 \pi \times 2 \pi$ cube, which is discretized with an homogeneous grid with periodic boundary conditions.

The flow is defined by the turbulent Mach number $\left(M_{t}\right)$ and Taylor's microscale Reynolds number $\left(R e_{\lambda}\right)$, which are defined as 


$$
\begin{aligned}
M_{t} & =\frac{\sqrt{\left\langle v_{i}^{\prime} v_{i}^{\prime}\right\rangle}}{\langle c\rangle} \\
R e_{\lambda} & =\frac{\langle\rho\rangle v_{\mathrm{rms}}^{\prime} \lambda}{\langle\mu\rangle}
\end{aligned}
$$

The symbol \langle\rangle refers to mean value and primes denote fluctuating variables. Moreover, we define the root mean square of the velocity $\left(v_{\mathrm{rms}}\right)$ as

$$
v_{\mathrm{rms}}=\sqrt{\frac{\left\langle v_{i}^{\prime} v_{i}^{\prime}\right\rangle}{3}}
$$

The initial three-dimensional kinetic energy spectrum is defined as

$$
E_{3 D} \sim k^{4} \exp \left[-2\left(\frac{k}{k_{0}}\right)^{2}\right]
$$

where $k$ is the magnitude of the wave number vector, and $k_{0}=4$ is the wavenumber at the peak of the spectrum. Using this initial energy spectrum, $\lambda_{0}=0.5 k_{0}$.

In this section we will solve two different configurations of this problem. The first case, referred henceforth as DEC1 corresponds with the case 6 of [60]. For the DEC1 case, initial velocity fluctuations are parametrized by the turbulent Mach number, and also by the fraction of energy in the dilatational part of the velocity, $\chi=0.2[62]$. The initial turbulent Mach number is taken as $M_{t, 0}=0.4$.

The initial density and temperature fields are given by

$$
\begin{gathered}
\left(\rho_{r m s}^{\prime}\right)^{2} /\langle\rho\rangle^{2}=0.032 \\
\left(T_{r m s}^{\prime}\right)^{2} /\langle T\rangle^{2}=0.005
\end{gathered}
$$

The initial Taylor's microscale Reynolds number is chosen as $R e_{\tau, 0}=2157$ which corresponds with a Reynolds number $R e=536.9$ [63]. 
The second configuration, henceforth referred as DEC2, is taken from $[18,21,32]$. In this case the density and pressure fields are initially constant (here we set $\rho=1$, and the pressure field is obtained accordingly), with $M_{t, 0}=0.6$ and $R e_{\lambda, 0}=100$ as initial parameters.

These setups correspond to the nonlinear subsonic regime [1] and weak shocklets develop spontaneously from the turbulent motion. This fact represents a challenge to the accuracy of any numerical scheme [18, 21, 32].

In the simulations of the decay problem, we have used an initial value of the the dissipation coefficient $\epsilon_{i n i}=0.15$. The reason is that a value of $\epsilon_{i n i}=1$ introduce excessive dissipation in the beginning of the simulation, that greatly affects the final results. We note that this is a specific problem of this test case, with a specific set of initial conditions. In regular practice of turbulent flow computations it is usual to let the flow develop before starting the simulation. In that case, the period of development of the flow is enough to adjust the dissipation coefficient.

\subsubsection{DEC1 case results}

We run this simulation using a time step of $\Delta t=0.05$ for the $32^{3}$ grid which corresponds to 150 time-steps per eddy turnover time $\tau$, that is $\tau=$ 12.5 for this configuration. For the $64^{3}$ grid computation we keep the CFL constant. The results obtained for the DEC1 configuration of the decay problem are shown in figures 14 and 16. Two different grids were used for the computations. A coarse grid of $32^{3}$ elements and a finer one of $64^{3}$. These grids correspond to under-resolved simulations, since we are interested in the behavior of the scheme for LES simulations. The obtained results are compared with a reference solution computed with a sixth-order compact 
finite difference scheme, with explicit filtering using a tenth-order Padé filter. This solution follows closely the results of a DNS [64].
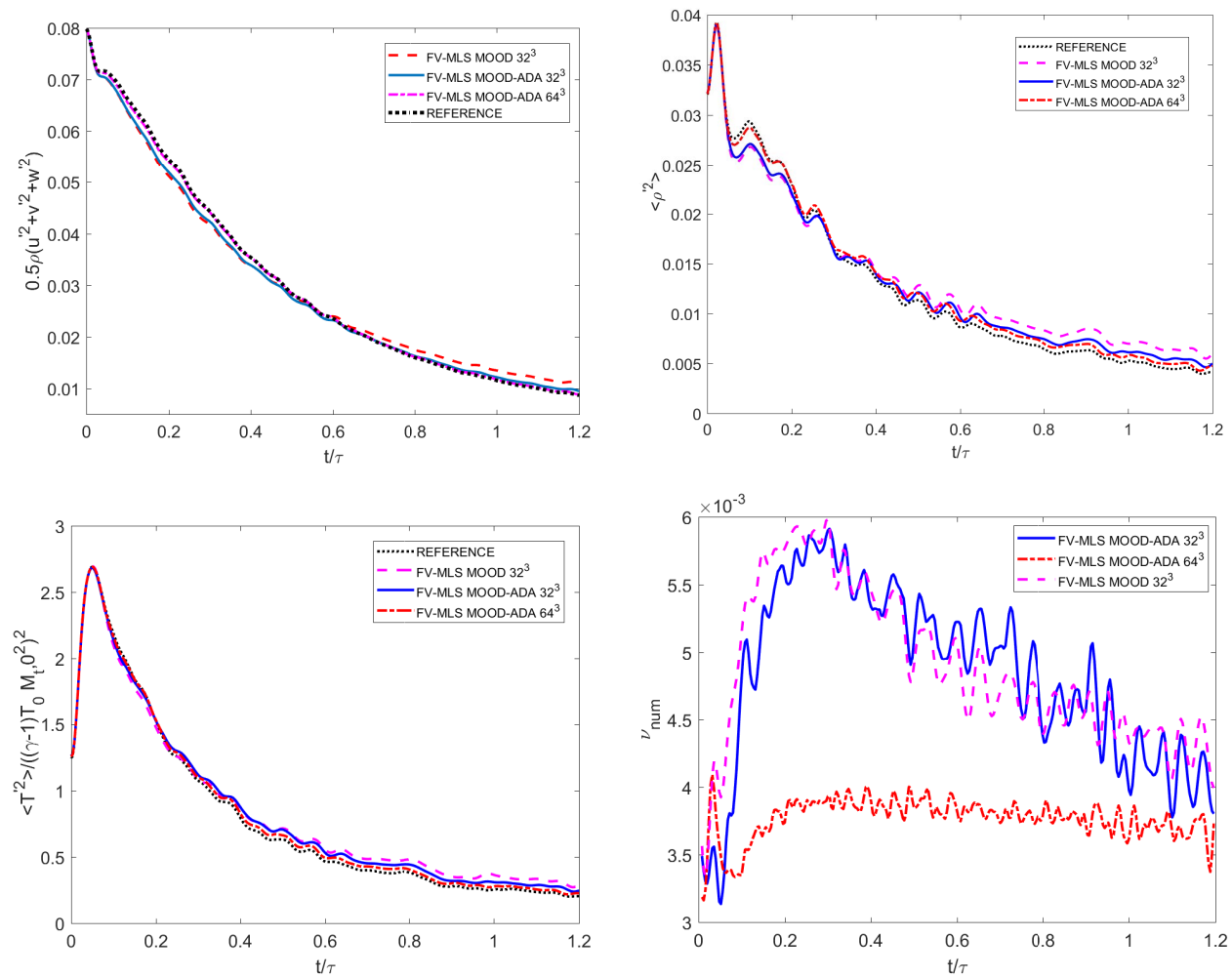

Figure 14: Decay of homogeneous isotropic turbulence. DEC1 Configuration. Evolution of the kinetic energy (top-left), mean-square density fluctuations (top-right), normalized mean-square temperature fluctuations (bottom-left) and evolution of the numerical viscosity (bottom-right), obtained with (indicated with ER in the legend) and without the proposed scheme, using a $32^{3}$ grid and compared with a reference solution [64]. The results obtained with the proposed scheme using a $64^{3}$ grid are also shown.

It is observed the general improvement obtained used the proposed scheme. Moreover, in figure 14 it is shown that the results converge to the reference solution as the grid is refined. Noteworthy, the scheme is able to reproduce 
the thermodynamic variables of the flow. This is important, since in [58] it is shown that some methods proposed for Implicit LES are not able to simultaneously predict the correct scaling and decay rates of thermodynamic variables.

The evolution of the numerical viscosity of the scheme with and without the proposed methodology is shown in figure 14. The numerical viscosity has been computed as proposed in [65]. It is shown that for values of $t / \tau=0$ to $t / \tau=0.4$, the proposed algorithm with adaptive viscosity is clearly less dissipative than the algorithm with fixed viscosity (that is, $\epsilon=1$ ). However, it is observed that from $t / \tau=0.4$ to $t / \tau=0.6$ the numerical viscosity of the adaptive viscosity algorithm is greater than that of the fixed viscosity algorithm. The reason of this behavior is similar to that already explained in the TGV test case, as can be seen examining the evolution of the kinetic energy. In figure 15 it is seen that the slope of the decay is bigger for the adaptive scheme than for the fixed viscosity scheme. Moreover, the slope of the adaptive scheme agrees with that of the reference solution. The largest amount of dissipation is introduced by the activation of the NAD. Since the solution is under resolved, the presence of numerical oscillations induces a reduction of the order in those cells, increasing the numerical dissipation. Note that the dynamics of the decay is completely different for each of the schemes, since the distribution of energy dissipation through the scales is different, as it can be confirmed examining the instantaneous three-dimensional energy spectra (see figure 16).

In order to compare with the results of $[63,64]$, the instantaneous threedimensional energy spectra for $\left.E(k)=\rho\left(u^{2}+v^{2}+w^{2}\right)\right)$ at $t / \tau=0.3$ is 


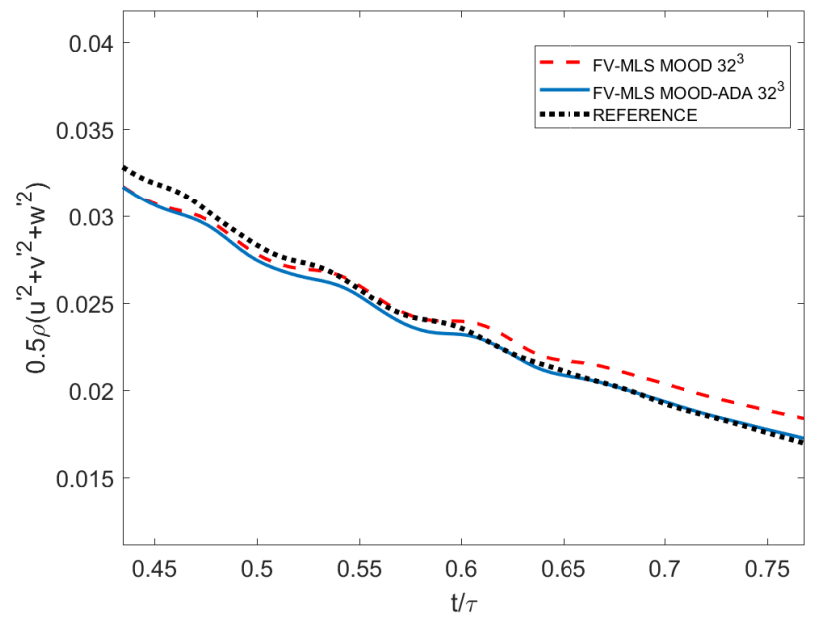

Figure 15: Decay of homogeneous isotropic turbulence. DEC1 Configuration. Zoom on the evolution of the kinetic energy for the $32^{3}$ (left) from $t / \tau=0.4$ to $t / \tau=0.6$.

plotted in figure 16. We note the two different slopes appearing in the energy spectrum, which agrees with the Eddy-Damped Quasi-Normal Markovian Theory (EDQNM) [66]. The obtained spectra using the proposed scheme fits almost perfectly the reference solution. The reduction in the dissipation of the smallest scales is clearly observed. This behavior holds as the grid is refined, as observed in the results for the $64^{3}$ grid. In both grids, no pile-up of energy is detected. These results confirm that the proposed methodology is a good candidate for Implicit LES computations.

The values of the dissipation coefficient $\epsilon$ at $t / \tau=0.3$ are shown in figure 17. Most of the points are in the range $0.1-0.6$, and, it is observed that the value $\epsilon=1$ is only reached in few zones of the whole computational domain. 

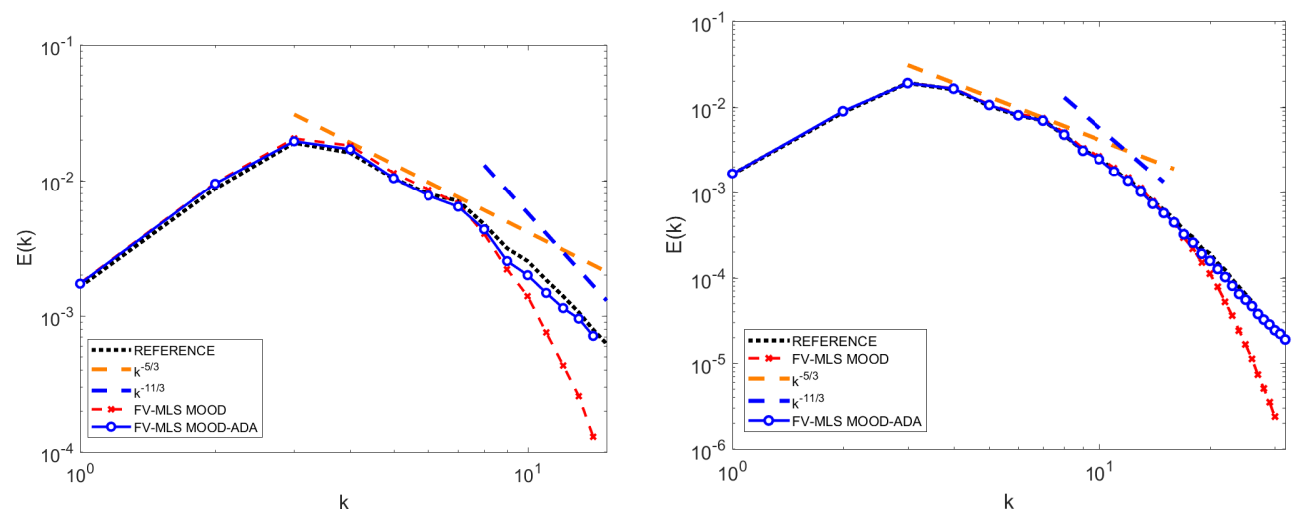

Figure 16: Decay of homogeneous isotropic turbulence. DEC1 Configuration. Instantaneous three-dimensional energy spectra at $t / \tau=0.3$. Results for the $32^{3}$ (left) and $64^{3}$ (right) grids.

\subsubsection{DEC2 case results}

The second configuration of the decay problem present stronger shocklets in the solution, and is a harder test case than the previous one. We run this simulation using a time step of $\Delta t=0.005$. In this case, the eddy turn-over time is $\tau=k_{0} / v_{r m s 0}=0.5$.

The results obtained with the proposed scheme are shown in figure 18. In order to compare with $[18,21,32]$, the evolution of the mean-square velocity, enstrophy, normalized mean-square temperature fluctuations and evolution of dilatation, obtained on a $64^{3}$ grid are shown. The reference solution is the DNS solution digitized from [18].

It is observed the excellent agreement between the results of the proposed scheme and the reference solutions for the mean-square velocity and temperature fluctuations. We note that although the dilatation and enstrophy results are somewhat over-dissipative, the results obtained are in the range of most of the higher-order methods compared in [18]. 

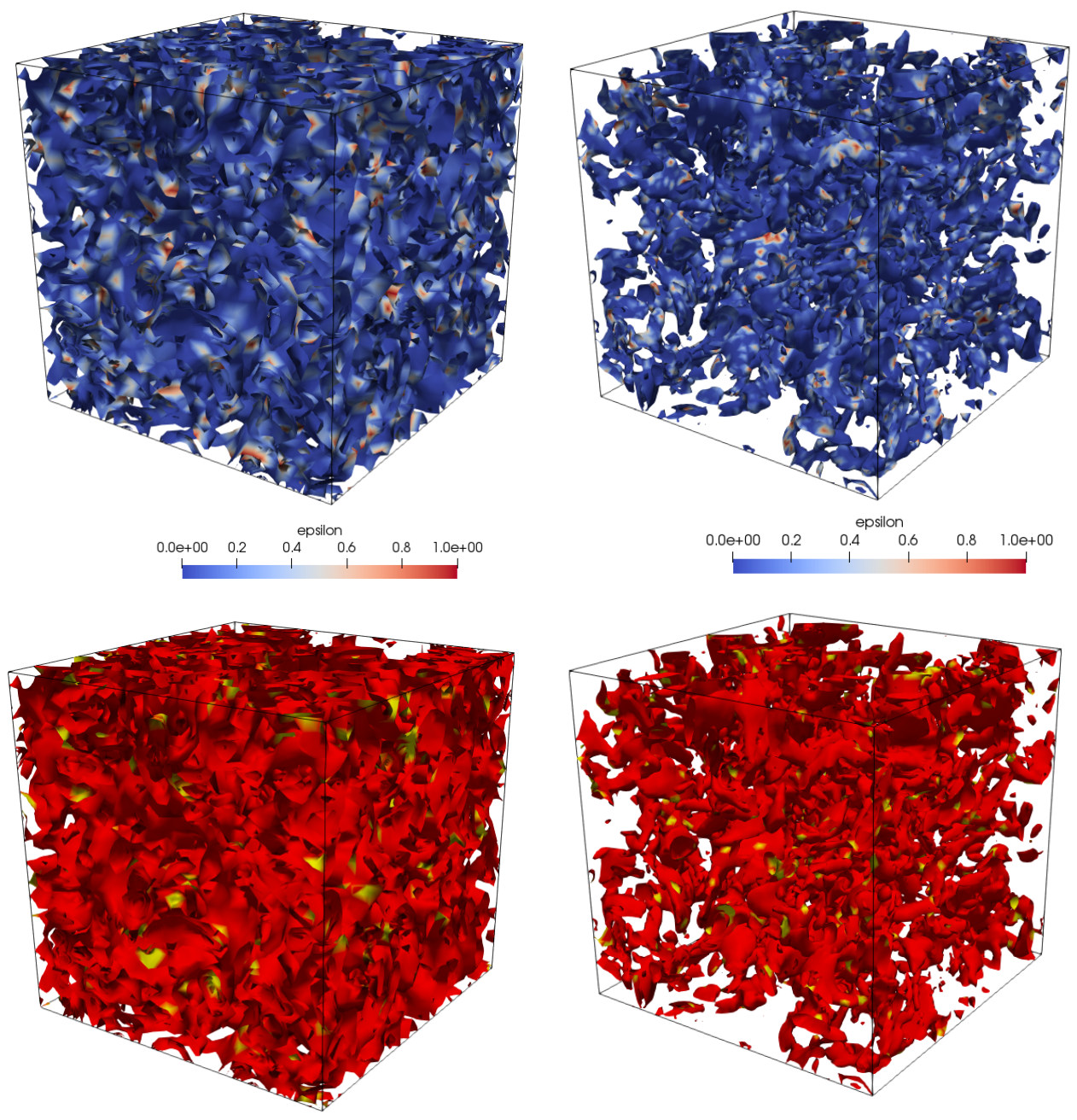

Figure 17: Decay of homogeneous isotropic turbulence. DEC1 Configuration: 0.2 absolute value of the vorticity iso-contours, colored with the instantaneous value of $\epsilon$ at $t / \tau=0.3$ on the $32^{3}$ grid (top-left) and $64^{3}$ grid (top-right). On the bottom the detected cells (in yellow) at $t / \tau=0.3$ on the $32^{3}$ grid (bottom-left) and $64^{3}$ grid (bottom-right) are shown.

The instantaneous three-dimensional velocity spectra $E_{u}(k)=u^{2}+v^{2}+w^{2}$ at the final time of the simulation $t / \tau=4$ is shown in figure 19. As in 

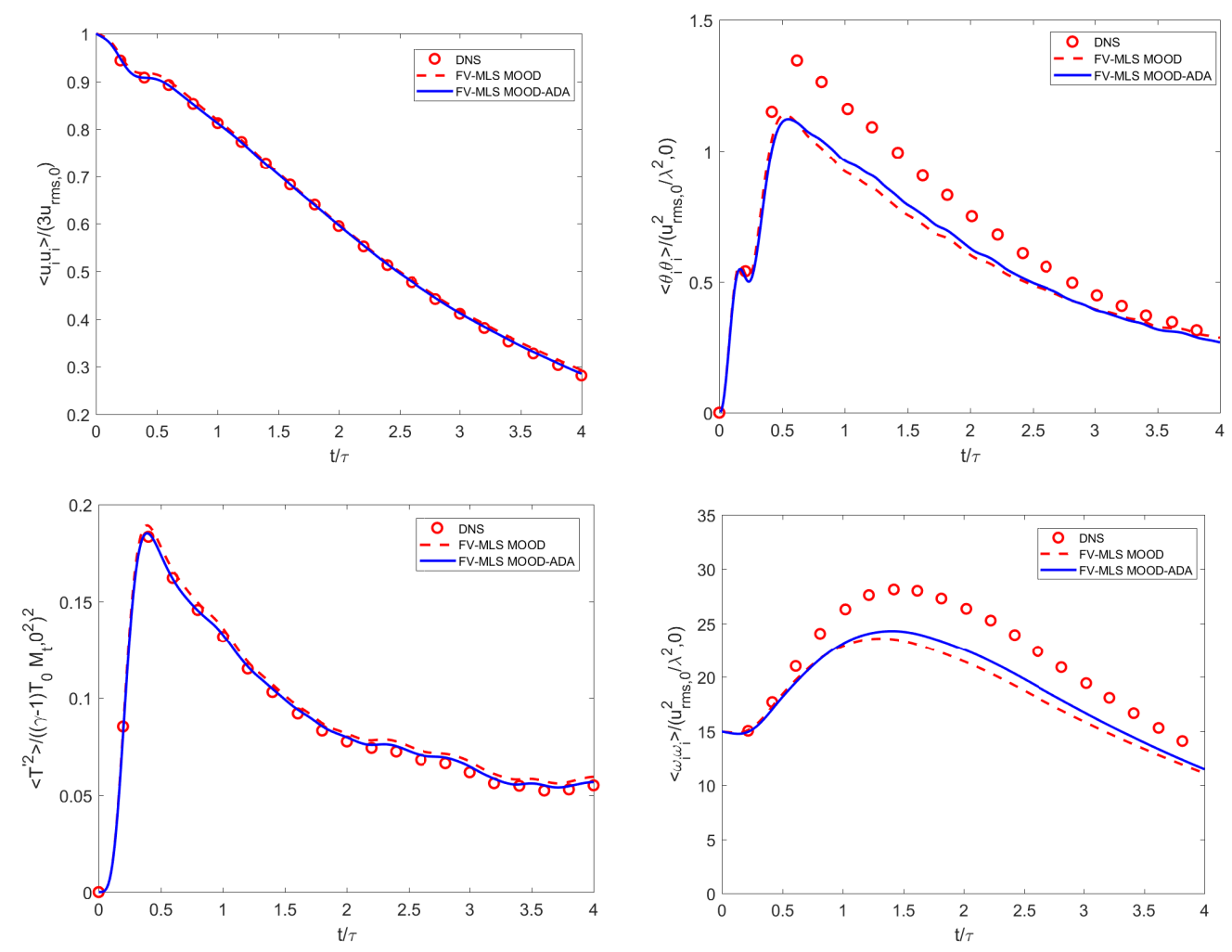

Figure 18: Decay of homogeneous isotropic turbulence. DEC2 Configuration. Evolution of the mean-square velocity (top-left), dilatation (top-right), normalized mean-square temperature fluctuations (bottom-left) and enstrophy (bottom-right), obtained with (indicated with ER in the legend) and without the proposed scheme, using a $64^{3}$ grid. The reference solution is the DNS solution digitized from [18].

the previous case, a very good agreement is observed between the proposed scheme and the DNS solution. Moreover, an improved accuracy is observed at the smallest scales, compared to the third-order FV-MLS scheme without the proposed method.

The values of the dissipation coefficient $\epsilon$ at $t / \tau=4$ are shown in figure 20. Most of the points are in the range $0-0.6$, and it is observed that the 


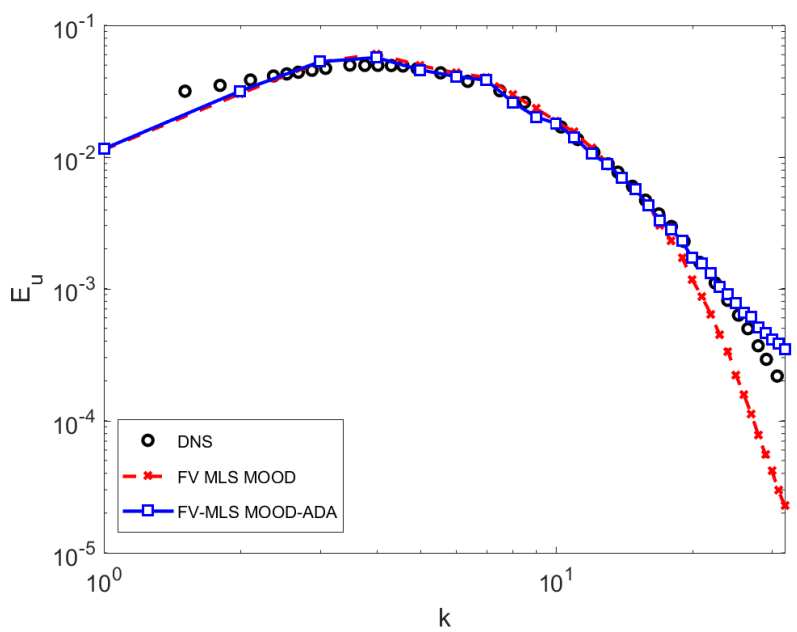

Figure 19: Decay of homogeneous isotropic turbulence. DEC2 Configuration. Instantaneous three-dimensional velocity spectra at $t / \tau=4$, obtained with (indicated with ER in the legend) and without the proposed scheme, using a $64^{3}$ grid. DNS results are digitized from $[18]$

value $\epsilon=1$ is only reached in few zones of the whole computational domain. In the same figure (right) the cells where the a posteriori detection criteria was activated are shown.

The total number of cells activated during each time step by the a posteriori algorithm is shown in figure 21 . At the beginning of the computation, the number of cells marked by the algorithm reachs its maximum, with around $48 \%$ of the total cells at the first iteration. However, the number of detected cells decreases very quickly, and it remains stable at around $0.5 \%$ for most of the computation time. It is seen that the number of cells to recompute is small, and thus, the additional dissipation introduced in order to stabilize the scheme is not excessive. Moreover, since the number of detected cells is small, the number of cells to be recomputed is also small, and the computa- 

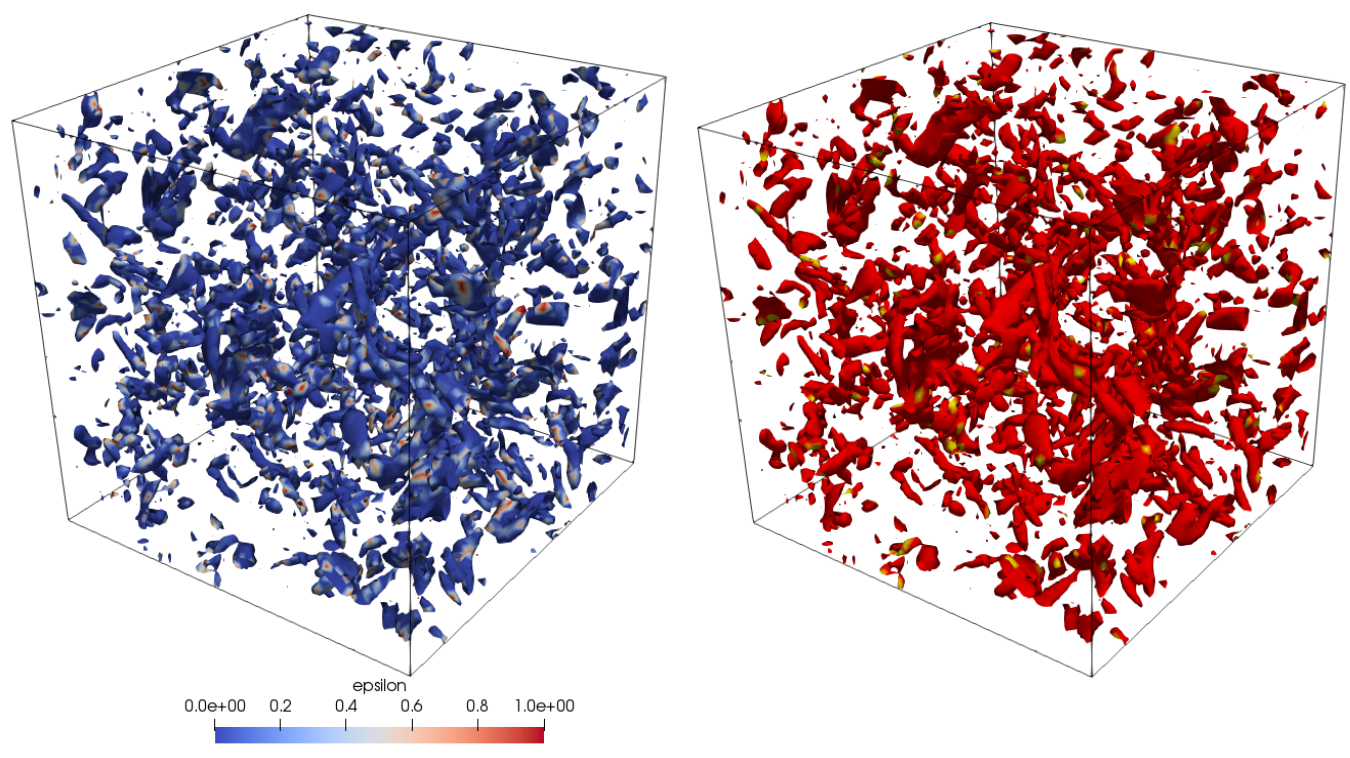

Figure 20: Decay of homogeneous isotropic turbulence. DEC2 Configuration. Q-criterion contours ( 8 contours from -2 to 1 ) colored with the instantaneous value of $\epsilon$ at $t / \tau=4$ (left) and detected cells (in yellow) with the NAD and PAD criteria (right).

tional cost of the proposed scheme is not greatly increased. This behavior of the a posteriori techniques has also been reported in [30].

\subsection{D Mach 3 wind tunnel with a step}

In order to address a case with stronger shocks, we solve here the 2D supersonic flow across a wind tunnel, as proposed in [67]. In this test case, a supersonic flow at Mach 3 across a wind tunnel of 1 length unit wide and 3 length units long is considered. A step is located at 0.6 length units from the inflow, and it is 0.2 length units wide. This is an inviscid case, in order to consider an infinite Reynolds number. We use slip wall boundary conditions along the walls, and supersonic inflow and outflow boundary conditions. The initial value of the velocity is set to $u=3, v=0$, whereas the initial values 


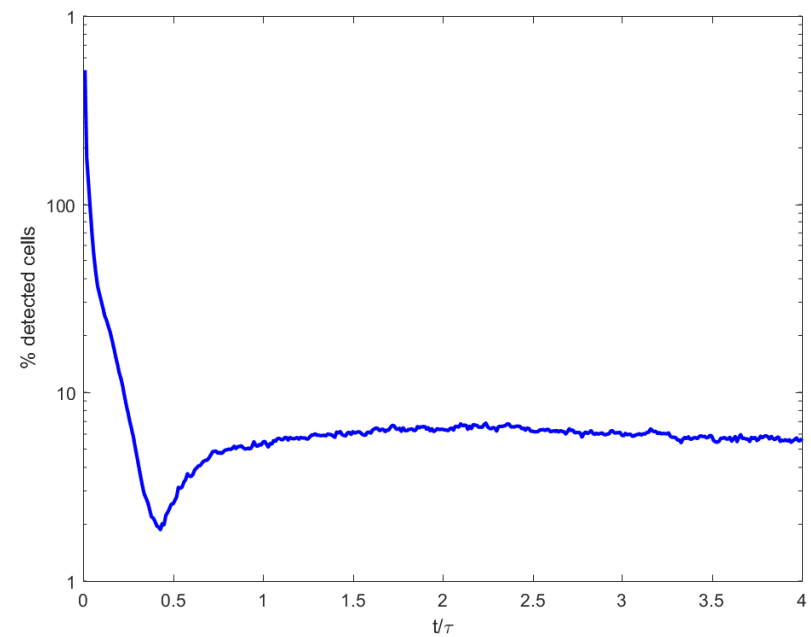

Figure 21: Decay of homogeneous isotropic turbulence. DEC2 Configuration. Troubled cells detected by the a posteriori algorithm.

of density and pressure are defined as $\rho=1.4, p=1$, and $\gamma=1$.4. This setup corresponds to a Mach 3 flow. Following [68], the singularity point at the corner is managed by refining the mesh in this region. The mesh is built by setting the biggest size of the elements away from the corner as $\Delta x=\Delta y=1 / 160$, and the size of elements near the corner is one-half that. A paving algorithm has been used to build the mesh. A detail of the grid is plotted in figure 22. We solve the two-dimensional Euler equations using the Rusanov scheme given in equation (16).

The results are shown in figures 23 and 24 . We show that the method is stable and it obtains very accurate results using the ADA method combined with the a posteriori approach. In particular, it is shown that, using the proposed method, we are able to reproduce the Kelvin-Helmholtz instability that forms after the shock. Using the same numerical discretization scheme 


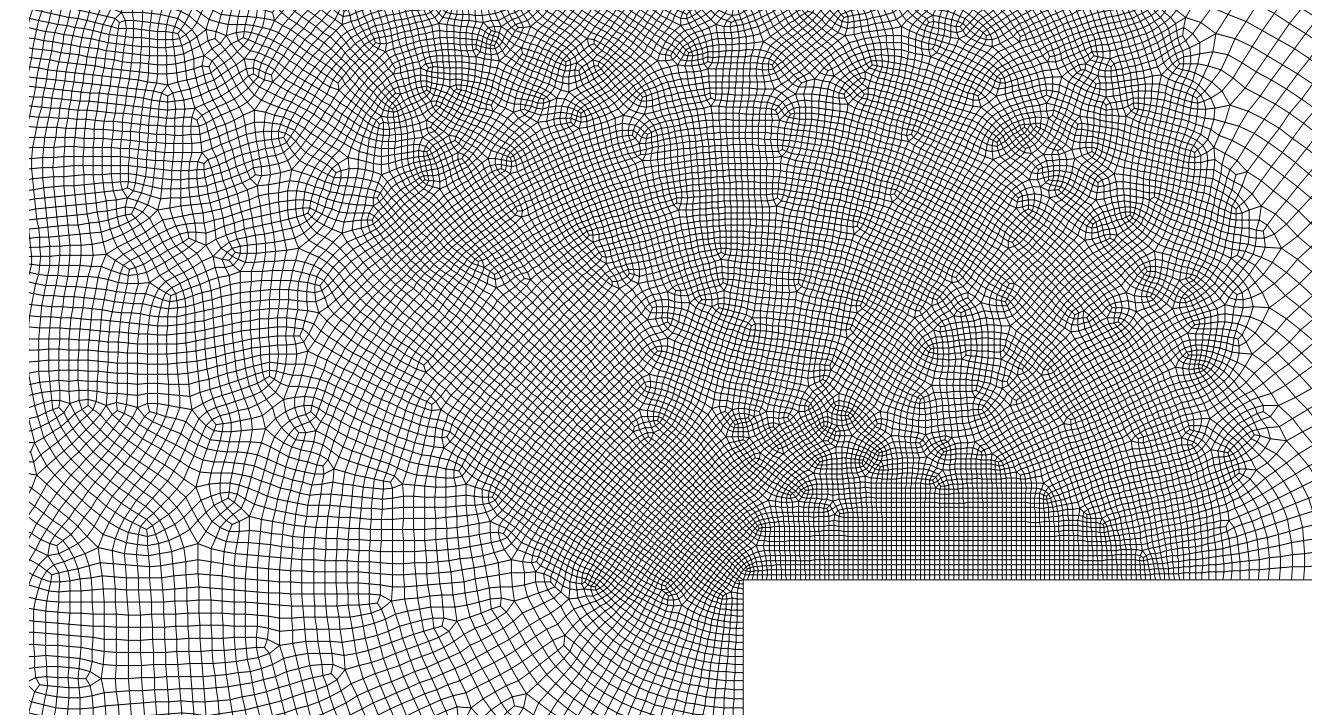

Figure 22: Mach 3 wind tunnel problem. Detail of the grid.

but without the $\mathrm{ADA}$ and the a posteriori detection, (that is, a classical approach based on slope limiters), the Kelvin-Helmholtz instability is not captured. Moreover, the Mach stem at the step wall, is much shorter using the proposed approach.

Figure 24 shows that in most of the domain, the value of $\epsilon$ is small, and that the number of cells with the reduced order is also small. The area where the Kelvin-Helmholtz instability develops is solved with the third-order scheme with reduced numerical viscosity.

\section{Conclusions}

We have presented an a posteriori high-order finite volume scheme using Roe and Rusanov numerical fluxes for the computation of compressible turbulent flows. An Implicit LES scheme has been proposed by combining an automatic dissipation adjustment (ADA) method with the a posteriori 


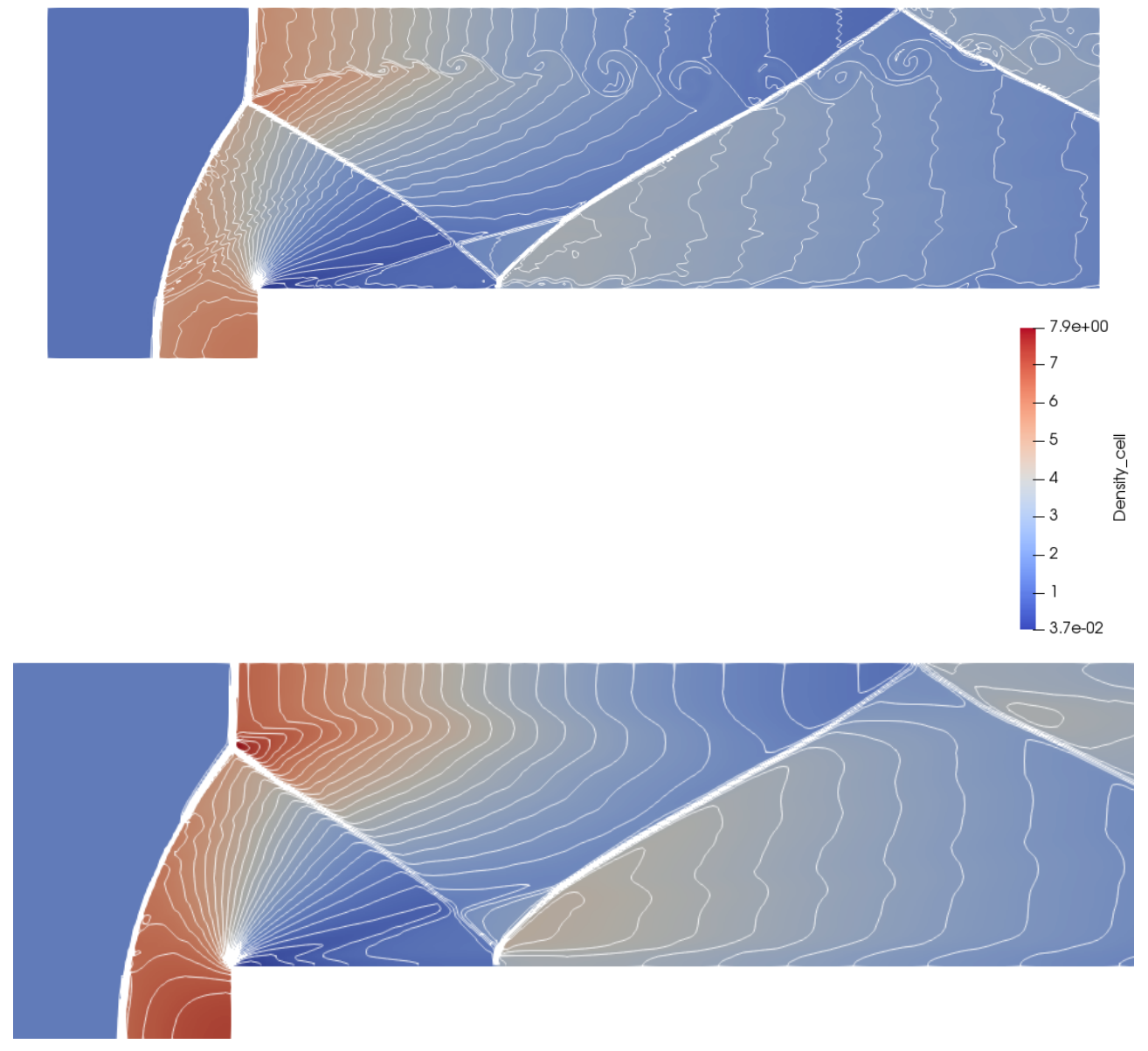

Figure 23: Mach 3 wind tunnel problem. Density contours from 0.212 to 6.22 at time $t=4$ contours obtained with the proposed approach (top) and using an approach based on slope limiters (bottom). 

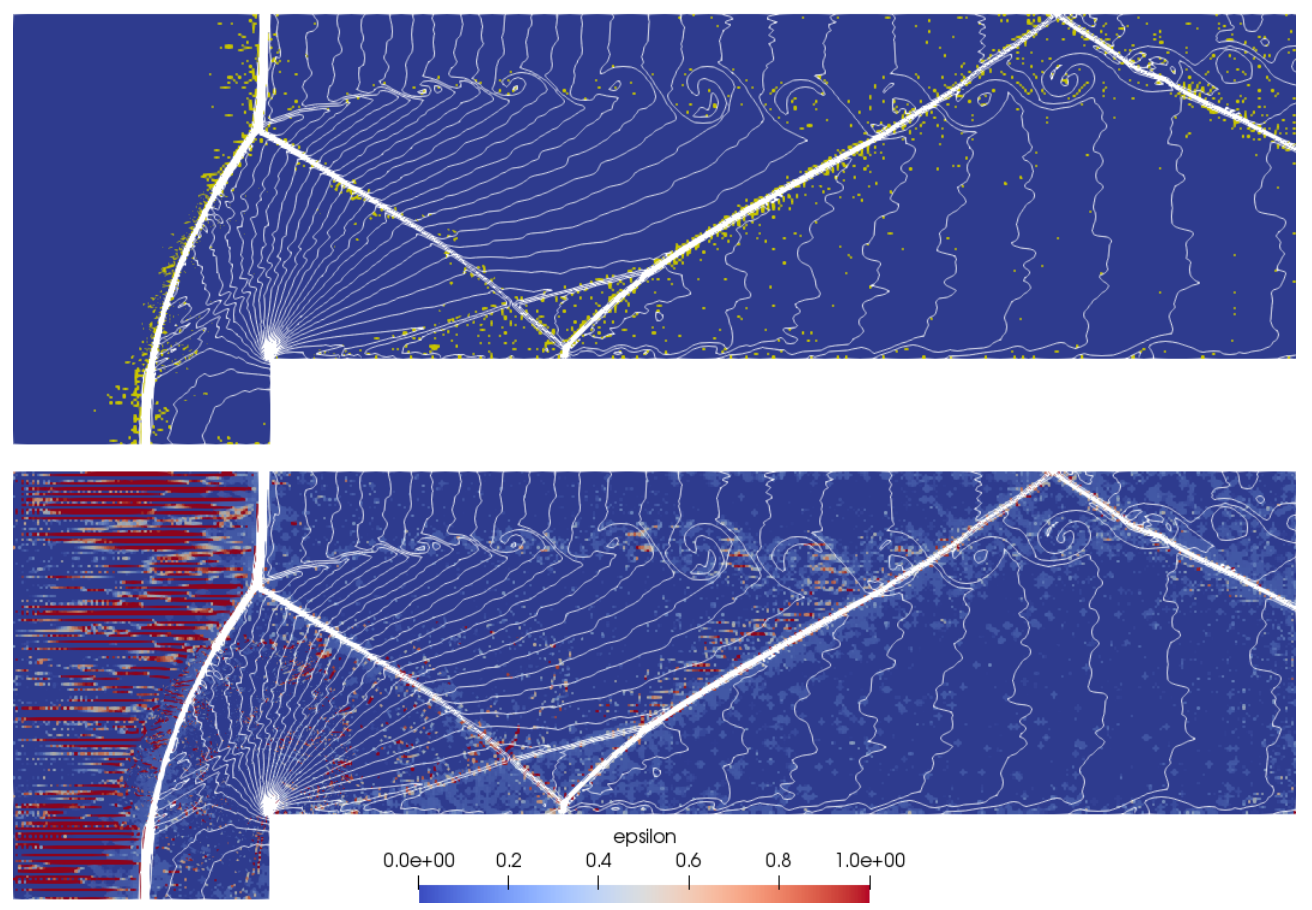

Figure 24: Mach 3 wind tunnel problem. Detected cells (in red) with the a posteriori approach (top) and value of $\epsilon$ (bottom). We also plot 30 density contours from 0.212 to 6.22 at time $t=4$.

paradigm. It has been demonstrated that the proposed methodology is able to implicitly define a subgrid scale model and it also keeps the stability of the computations. The numerical dissipation is adjusted by adding a multiplicative factor to the dissipative part of the numerical flux. The a posteriori approach allows for detecting the cells where the stability is compromised, and thus where dissipation must be added to ensure the stability of the scheme. Through the Taylor-Green vortex and the isotropic decay of turbulence test cases, we shown that the proposed methodology is a promising candidate for ILES simulations, since the physics of the decay is reproduced by our simulations. The proposed method is able to obtain comparable results to those 
obtained using numerical methods with higher order of accuracy. Moreover, the Mach 3 step flow case shows the accuracy and robustness of the the proposed numerical scheme, which is capable to work with shock waves of considerable strength. We note that the proposed methodology presents a way to greatly improve the accuracy of existing second or third-order finite volume codes. This work is a first step in the development of a complete ILES methodology for compressible flows. However, further research is required for non-isotropic turbulence, particularly for wall-bounded compressible turbulent flows using unstructured grids.

\section{Acknowledgments}

The authors greatly acknowledge the kind help of Dr. D. P. Rizzeta, Dr. Lin $\mathrm{Fu}$ and Dr. H. C. Yee, who kindly share with us the initial conditions for the turbulence decay problems.

This work has been partially supported by FEDER funds of the European Union, Grants \#DPI2015-68431-R of the Ministerio de Economía y Competitividad and\#RTI2018-093366-B-I00 of the Ministerio de Ciencia, Innovación y Universidades of the Spanish Government, by the Consellería de Cultura, Educación e Ordenación Universitaria of the Xunta de Galicia (program Axudas para potenciación de grupos de investigación do Sistema Universitario de Galicia 2018, grant \# ED431C 2018/41) and Research fellowships of the Universidade da Coruña and the Fundación de la Ingeniería Civil de Galicia. 
[1] P. Sagaut, Large Eddy Simulation for Compressible Flows. Berlin: Springer Verlag, ISBN: 978-90-481-2818-1, 2009.

[2] V. Venkatakrishnan, Convergence to steady state solutions of the Euler equations on unstructured grids with limiters, Journal of Computational Physics, 118:120-130, 1995.

[3] P. Tsoutsanis, Extended bounds limiter for high-order finite-volume schemes on unstructured meshes, Journal of Computational Physics, 362:69-94, 2018.

[4] X. Nogueira, L. Ramírez, S. Khelladi, J-C. Chassaing, I. Colominas, A high-order density-based finite volume method for the computation of allspeed flows, Computer Methods in Applied Mechanics and Engineering, 298:229-251, 2016.

[5] J.P. Boris, On large eddy simulation using subgrid turbulence models, Whither Turbulence? Turbulence at the crossroads, J. L. Lumley (Ed.), New York: Springer-Verlag, 344-353, 1990.

[6] J.P. Boris, F.F. Grinstein, E.S. Oran, R.S. Kolbe, New insights into Large Eddy Simulation, Fluid Dynamic Research, 10:199-228, 1992.

[7] C. Fureby, F.F. Grinstein, Monotonically Integrated large eddy simulation of free shear flows, AIAA journal, 37(5):544-556, 1999.

[8] C. Fureby, F.F. Grinstein, Large Eddy Simulation of High-Reynolds- 
Number Free and and Wall-Bounded Flows, Journal of Computational Physics, $181: 68-97,2002$.

[9] F.F. Grinstein, C. Fureby, Recent Progress on MILES for high Reynolds Number flows, Journal of Fluid Engineering, 124:848-861, 2002.

[10] D. Drikakis, M. Hahn, A. Mosedale, B. Thornber, Large eddy simulation using high-resolution and high-order methods, Philosophical Transactions of the Royal Society A, 367:2985-2997, 2009.

[11] A. Uranga, P.O. Persson, M. Drela, J. Peraire, Implicit Large Eddy Simulation of transition to turbulence at low Reynolds numbers using a Discontinuous Galerkin method, International Journal of Numerical Methods in Engineering, 87: 232-261, 2011.

[12] T.J.R. Hughes, A.A. Oberai, L. Mazzei, Large-eddy simulation of turbulent channel flows by the variational multiscale method, Physics of Fluids, 13:1784-1799, 2001.

[13] X. Nogueira, L. Cueto-Felgueroso, I. Colominas, H. Gómez, Implicit Large Eddy Simulation of non-wall-bounded turbulent flows based on the multiscale properties of a high-order finite volume method, Computer Methods in Applied Mechanics and Engineering, 199:315-624, 2010.

[14] X. Nogueira, S. Khelladi, I. Colominas, L. Cueto-Felgueroso, J. París, High-Resolution Finite Volume Methods on Unstructured Grids for Turbulence and Aeroacoustics, Archives of Computational Methods in Engineering, 18(3):315-340, 2011. 
[15] E. Garnier, M. Mossi, P. Sagaut, P. Comte, M. Deville, On the use of shock-capturing schemes for large-eddy simulation, Journal of Computational Physics, 153(2):273-311, 1999.

[16] S. Hickel, N.A. Adams, J.A. Domaradzki, An adaptive local deconvolution method for implicit LES, Journal of Computational Physics, 213(1):413-436, 2006.

[17] C.P. Egerer, S.J. Schmidt, S. Hickel, N.A. Adams, Efficient implicit LES method for the simulation of turbulent cavitating flows, Journal of Computational Physics, 316:453-469, 2016.

[18] E. Johnsen, J. Larsson, A.V. Bhagatwala, W.H. Cabot, P. Moin, B.J. Olson, P. S. Rawat, S.K. Shankar, B. SjÃúgreen, H.C. Yee, X. Zhong, S.K. Lele, Assessment of high-resolution methods for numerical simulations of compressible turbulence with shock waves, Journal of Computational Physics, 229:1213-1237, 2010.

[19] H.C. Yee, A.A. Wray, B. SjÃúgreen, A. Hadjadj, Comparative Study of Three High Order Schemes for LES of Temporally Evolving Mixing Layers, Communications in Computational Physics, 12(5):1603-1622, 2012.

[20] H.C. Yee, B. SjÃúgreen, in: Proceedings of ICOSAHOM 09, Trondheim, Norway, 2013.

[21] D.V. Kotov, H.C. Yee, A.A. Wray, B. SjÃúgreen, A.G. Kritsuk, Numerical dissipation control in high order shock-capturing schemes for LES of low speed flows, Journal of Computational Physics, 307:189-202, 2016. 
[22] E.F. Toro, Riemann Solvers and Numerical Methods for Fluid Dynamics. A Practical Introduction, Third edition. Berlin: Springer Verlag, ISBN: 978-3-540-25202-3, 1999.

[23] P.L. Roe, Approximate Riemann solvers, parameter vectors and difference schemes, Journal of Computational Physics, 43, 357-372, 1981.

[24] B. Thornber, A. Mosedale, D. Drikakis, D. Youngs, R.J.R. Williams, An improved reconstruction method for compressible flows with low Mach number features, Journal of Computational Physics, 22\%:4873-4894, 2008.

[25] F. Rieper, A low-Mach number fix for Roe's approximate Riemann solver, Journal of Computational Physics, 230:5263-5287, 2011.

[26] M. Ciardi, P. Sagaut, M. Klein, W.N. Dawes, A dynamic finite volume scheme for large-eddy simulation on unstructured grids, Journal of Computational Physics, 210:632-655, 2005.

[27] N. Tajallipour, B.B. Owlam, M. Paraschivou Self-adaptive upwind for large eddy simulation of turbulent flows on unstructured elements, Journal of Aircraft, 46 (3):915-926, 2009.

[28] C-G Li, M. Tsubokura, An implicit turbulence model for low-Mach Roe scheme using truncated Navier-Stokes equations, Journal of Computational Physics, 345:462-474, 2017.

[29] T. Tantikul, J.A. Domaradzki,Large eddy simulations using truncated Navier-Stokes equations with the automatic filtering criterion, Journal of Turbulence, $11: 1-24,2010$. 
[30] S. Clain, S. Diot, R. Loubère A high-order finite volume method for systems of conservation laws- Multidimensional Optimal Order Detection (MOOD), Journal of Computational Physics, 230:4028-4050, 2011.

[31] S. Diot, S. Clain, R. Loubère, Improved detection criteria for the Multidimensional Optimal Order Detection (MOOD) on unstructured meshes with very high-order polynomials, Computers and Fluids, 64:43-63, 2012.

[32] L. Fu, X.Y. Hu and N. A. Adams, Implicit large eddy simulations with a high-order TENO scheme, 10th International Symposium on Turbulence and Shear Flow Phenomena, TSFP 2017.

[33] L. Fu, X. Hu, N.A. Adams, A targeted eno scheme as implicit model for turbulent and genuine subgrid scales, Communications in Computational Physics.

[34] L. Cueto-Felgueroso, I. Colominas, X. Nogueira, F. Navarrina, M. Casteleiro, Finite volume solvers and Moving Least-Squares approximations for the compressible Navier-Stokes equations on unstructured grids, Computer Methods in Applied Mechanics and Engineering, 196:4712-4736, 2007.

[35] X. Nogueira, L. Cueto-Felgueroso, I. Colominas, S. Khelladi, On the simulation of wave propagation with a higher-order finite volume scheme based on Reproducing Kernel Methods, Computer Methods in Applied Mechanics and Engineering, 199 (23-24):1471-1490, 2010.

[36] S. Khelladi, X. Nogueira, F. Bakir, I. Colominas, Toward a Higher Order Unsteady Finite Volume Solver Based on Reproducing Kernel Methods, 
Computer Methods in Applied Mechanics and Engineering, 200:2348-2362, 2011.

[37] J-.C. Chassaing, S. Khelladi, X. Nogueira, Accuracy assessment of a high-order moving least squares finite volume method for compressible flows, Computers \& Fluids, 71: 41-53, 2013.

[38] V.V. Rusanov, The calculation of the interaction of non-stationary shock waves and obstacles, USSR Computational Mathematics and Mathematical Physics, 1(2):304-320, 1962.

[39] J.A. Domaradzki, K.C. Loh, P.P. Yee, Large eddy simulations using the subgrid-scale estimation model and truncated Navier-Stokes dynamics, Theoretical and Computational Fluid Dynamics, 15:421-450, 2002.

[40] K. Masatsuka, I do like CFD, Vol. 1, no. v. 1, second ed., K. Masatsuka, 2013.

[41] F. Cadieux, J.A. Domaradzki,Periodic filtering as a subgrid-scale model for LES of laminar separation bubble flows, Journal of Turbulence, 17:954965, 2016.

[42] G.J. Wagner and W.K. Liu, Turbulence simulation and multiple scale subgrid models, Computational Mechanics, 25:117-136, 2000.

[43] X. Nogueira, L. Cueto-Felgueroso, I. Colominas, F. Navarrina, M. Casteleiro, A new shock-capturing technique based on Moving Least Squares for higher-order numerical schemes on unstructured grids, Computer Methods in Applied Mechanics and Engineering, 199:2544-2558, 2010. 
[44] G. Sun, J.A. Domaradzki, Implicit LES using adaptive filtering, Journal of Computational Physics, 359:380-408, 2018.

[45] M. Dumbser, O. Zanotti, R. Loubère and S. Diot, A posteriori subcell limiting of the discontinuous Galerkin finite element method for hyperbolic conservation laws, Journal of Computational Physics, 278: 47-75, 2014.

[46] R.N. Hollyer, O. Laporte, Parameters Characterizing the Strength of a Shockwave, American Journal of Physics, 21, 610,1953.

[47] C-W. Shu, S. Osher, Efficient implementation of essentially nonoscillatory shock capturing schemes, Journal of Computational Physics, 77\%: 439-471, 1988.

[48] J. Li, Q. Li, K. Xu, Comparison of the generalized Riemann solver and the gas-kinetic scheme for inviscid compressible flow simulations, Journal of Computational Physics, 230(12): 5080-5099, 2011.

[49] E. F. Toro, NUMERICA. A Library of Source Codes for Teaching, Research and Applications, https://eleuteriotoro.com/software/

[50] S.E. Sherer, J. N. Scott, High-order compact finite-difference methods on general overset grids. Journal of Computational Physics, 210: 459496, 2005.

[51] L. Ramirez, C. Foulquié, X. Nogueira, S. Khelladi, J-.C. Chassaing and I. Colominas, New high-resolution-preserving sliding mesh techniques for higher-order finite volume schemes, Computers \& Fluids, 118: 114-130, 2015 . 
[52] L. Ramirez, X. Nogueira, P. Ouro, F. Navarrina, S. Khelladi and I. Colominas, A Higher-Order Chimera Method for Finite Volume Schemes,Archives of Computational Methods in Engineering, 25(3):691706, 2018.

[53] D. Fauconnier, C. De Langhe, E. Dick, Construction of explicit and implicit dynamic finite difference schemes and application to the large-eddy simulation of the Taylor-Green vortex, Journal of Computational Physics, 228(21): 8053-8084, 2009.

[54] C-W. Shu, W-S. Don, D Gottlieb, O. Schilling and L. Jameson, Numerical Convergence Study of Nearly Incompressible, Inviscid Taylor-Green Vortex Flow, Journal of Scientific Computing, 24(1):1-27, 2005.

[55] K.Grimich, P.Cinnella, A.Lerat, Spectral properties of high-order residual-based compact schemes for unsteady compressible flows, Journal of Computational Physics 252:142-162, 2013.

[56] M.E. Brachet, D.I. Meiron, S.A. Orszag, B.G. Nickel, R.H. Morf, U. Frisch, Small-scale structure of the Taylor-Green vortex, Journal of Fluid Mechanics, 130: 411-452, 1983.

[57] C. Carton de Wiart, K. Hillewaert, L. Bricteux and G. Winckelmans, Implicit LES of free and wall-bounded turbulent flows based on the discontinuous Galerkin/symmetric interior penalty method, International Journal for Numerical Methods in Fluids, 78(6):335-354, 2015.

[58] A.E. Honein, P. Moin, Higher entropy conservation and numerical sta- 
bility of compressible turbulence simulations, Journal of Computational Physics, 201:531-545, 2004.

[59] S. Lee, S.K. Lele, and P. Moin, Eddy shocklets in decaying compressible turbulence, Physics of Fluids A: Fluid Dynamics (1989-1993) 3:657, 1991.

[60] E.T. Spyropoulos, G.A. Blaisdell, Evaluation of the Dynamic Model for Simulations of Compressible Decaying Isotropic Turbulence, AIAA Journal, $34(5): 990-998,1996$

[61] S. Hickel, C.P. Egerer, J. Larsson, Subgrid scale modeling for implicit large eddy simulation of compressible flows and shock turbulence interaction, Physics of Fluids, 26:106101, 2014.

[62] S. Sarkar, G. Erlebacher, M.Y. Hussaini, H.O. Kreiss, The analysis and modeling of dilatational terms in compressible turbulence, Journal of Fluid Mechanics, 227:473-493, 1991.

[63] D.P. Rizzeta, M.R. Visbal and G.A. Blaisdell, Application of a Highorder Compact Difference Scheme to Large-Eddy and Direct Numerical Simulation, A99-33703, 31th AIAA Fluid Dynamics Conference, Norfolk, VA, 1999.

[64] M.R. Visbal and D.P. Rizzeta,Large-Eddy simulation on Curvilinear Grids Using Compact Differencing and Filtering Schemes, Journal of Fluids Engineering, 124:836-847, 2002.

[65] F.S. Schranner, J.A. Domaradzki, S. Hickel, N.A. Adams, Assessing the numerical dissipation rate and viscosity in numerical simulations of fluid flows, Computers and Fluids, 114, 84-97, 2015. 
[66] M.Y. Hussaini, On Large-Eddy Simulation of Compressible Flows, AIAA 29th Fluid Dynamics Conference, Albuquerque, New Mexico, Paper AIAA 98-2802, 1998.

[67] P. Woodward, P. Colella, The Numerical Simulation of Two-Dimensional Fluid Flow with Strong Shocks, Journal of Computational Physics, 54, 115$173,1984$.

[68] C.Q. Hu, C-W. Shu, Weighted essentially non-oscillatory schemes on triangular meshes, Journal of Computational Physics, 150, 97-127, 1999. 